Pacific

Journal of

Mathematics

\title{
REMARKS ON THE BEHAVIOR OF NONPARAMETRIC CAPILLARY SURFACES AT CORNERS
}

\author{
KIRK E. LANCASTER
}




\title{
REMARKS ON THE BEHAVIOR OF NONPARAMETRIC CAPILLARY SURFACES AT CORNERS
}

\author{
KIRK E. LANCASTER
}

\begin{abstract}
Consider a nonparametric capillary or prescribed mean curvature surface $z=f(x)$ defined in a cylinder $\Omega \times \mathbb{R}$ over a two-dimensional region $\Omega$ whose boundary has a corner at $\mathcal{O}$ with an opening angle of $2 \alpha$. Suppose the contact angle approaches limiting values $\gamma_{1}$ and $\gamma_{2}$ in $(0, \pi)$ as 0 is approached along each side of the opening angle. We will prove the nonconvex ConcusFinn conjecture, determine the exact sizes of the radial limit fans of $f$ at $\mathcal{O}$ when $\left(\gamma_{1}, \gamma_{1}\right) \in D_{1}^{ \pm} \cup D_{2}^{ \pm}$and discuss the continuity of the Gauss map.
\end{abstract}

\section{Introduction}

Let $\Omega \subset \mathbb{R}^{2}$ be a connected, open set. Consider the classical capillary problem in a cylinder

$$
\begin{aligned}
N f & =\kappa f+\lambda \quad \text { in } \Omega, \\
T f \cdot v & =\cos \gamma \quad \text { (a.e.) on } \partial \Omega,
\end{aligned}
$$

and, more generally, the prescribed mean curvature problem in a cylinder

$$
\begin{aligned}
& N f=H(\cdot, f(\cdot)) \quad \text { in } \Omega, \\
& T f \cdot v=\cos \gamma \quad \text { (a.e.) on } \partial \Omega \text {, }
\end{aligned}
$$

where $T f=\nabla f / \sqrt{1+|\nabla f|^{2}}, N f=\nabla \cdot T f, v$ is the exterior unit normal on $\partial \Omega, H(x, t)$ is a weakly increasing function of $t$ for each $x \in \Omega$ and $\gamma=\gamma(x)$ is in $[0, \pi]$. We will let $\mathscr{Y}_{f}$ denote the closure in $\mathbb{R}^{3}$ of the graph of $f$ over $\Omega$. When $H(x, t)=\kappa t+\lambda$ (i.e., $f$ satisfies (1)-(2)) with $\kappa$ and $\lambda$ constants such that $\kappa \geq 0$, then the surface $\mathscr{Y}_{f} \cap(\Omega \times \mathbb{R})$ represents the stationary liquid-gas interface formed by an incompressible fluid in a vertical cylindrical tube with cross-section $\Omega$ in a microgravity environment or in a downward-oriented gravitational field, the subgraph $U=\{(x, t) \in \Omega \times \mathbb{R}: t<f(x)\}$ represents the fluid filled portion of the cylinder and $\gamma(x)$ is the angle (within the fluid) at which the liquid-gas interface meets the vertical cylinder at $(x, f(x))$; Paul Concus and Robert Finn have made

MSC2010: primary 76B45, 35J93; secondary 53A10, 35J62.

Keywords: capillary graph, Concus-Finn conjecture, Gauss map, sizes of fans. 
fundamental contributions to the mathematical theory of capillary surfaces and have discovered that capillary surfaces can behave in unexpected ways (cf. [Concus and Finn 1996; Finn 1986; 1999; 2002b; 2002a]). For a function $f \in C^{2}(\Omega)$, we let

$$
\vec{n}(X)=\vec{n}_{f}(X)=\frac{(\nabla f(x),-1)}{\sqrt{1+|\nabla f(x)|^{2}}}, \quad X=(x, t) \in \Omega,
$$

denote the downward unit normal to the graph of $f$; when $f$ is a solution of (1)-(2) and $\kappa \geq 0, \vec{n}$ represents the inward unit normal with respect to the fluid region. Of interest here is the behavior of capillary surfaces and prescribed mean curvature surfaces over domains $\Omega \subset \mathbb{R}^{2}$ whose boundaries contain corners (e.g., [Concus and Finn 1996; Finn 1996]).

Let us suppose $O=(0,0) \in \partial \Omega$ and $\Omega$ is a connected, simply connected open set in $\mathbb{R}^{2}$ such that $\partial \Omega \backslash\{\mathcal{O}\}$ is a piecewise $C^{1}$ curve, $\Omega$ has a corner of size $2 \alpha$ at $O$ and the tangent cone to $\partial \Omega$ at $O$ is $L^{+} \cup L^{-}$, where polar coordinates relative to 0 are denoted by $r$ and $\theta, L^{+}=\{\theta=\alpha\}$ and $L^{-}=\{\theta=-\alpha\}$. We will assume there exist $\delta^{*}>0, \rho^{*} \in(0,1]$ such that $\partial^{+} \Omega=\partial \Omega \cap \overline{B\left(\mathcal{O}, \delta^{*}\right)} \cap T^{+}$and $\partial^{-} \Omega=\partial \Omega \cap \overline{B\left(\mathbb{O}, \delta^{*}\right)} \cap T^{-}$are connected, $C^{1, \rho^{*}}$ arcs, where $T^{+}=\left\{x \in \mathbb{R}^{2}: x_{2} \geq 0\right\}$, $T^{-}=\left\{x \in \mathbb{R}^{2}: x_{2} \leq 0\right\}$ and $B(\mathbb{O}, \epsilon)=\left\{x \in \mathbb{R}^{2}:|x|<\epsilon\right\}$; hence the tangent rays to $\partial^{+} \Omega$ and $\partial^{-} \Omega$ at $\mathcal{O}$ are $L^{+}$and $L^{-}$respectively. Set $\Omega_{0}=\Omega_{0}\left(\delta^{*}\right)=\Omega \cap B\left(\mathcal{O}, \delta^{*}\right)$. Let $\gamma^{+}(s)$ and $\gamma^{-}(s)$ denote $\gamma$ along the arcs $\partial^{+} \Omega_{0}$ and $\partial^{-} \Omega_{0}$, respectively, where $s=0$ corresponds to the point $\mathcal{O}$; here we have parametrized $\partial^{+} \Omega_{0}$ and $\partial^{-} \Omega_{0}$ by, for example, arclength $s$ from 0 and write these parametrizations as $x^{+}$and $x^{-}$ respectively. We will assume there exist $\gamma_{1}, \gamma_{2} \in(0, \pi)$ such that

$$
\lim _{\partial^{+} \Omega \ni x \rightarrow 0} \gamma(x)=\gamma_{1} \quad \text { and } \quad \lim _{\partial^{-} \Omega \ni x \rightarrow 0} \gamma(x)=\gamma_{2} .
$$

Suppose first that $2 \alpha \leq \pi$ (i.e., the corner is convex or $\partial \Omega$ is $C^{1}$ at 0 ); such an $\Omega$ is illustrated in Figure 1. Figure 2 can then be used to illustrate our knowledge

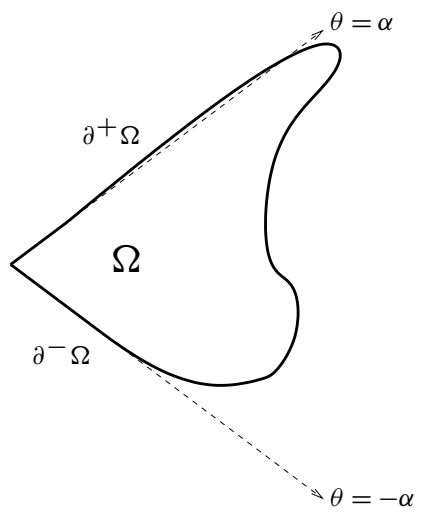

Figure 1. $\Omega$ with $2 \alpha<\pi$. 


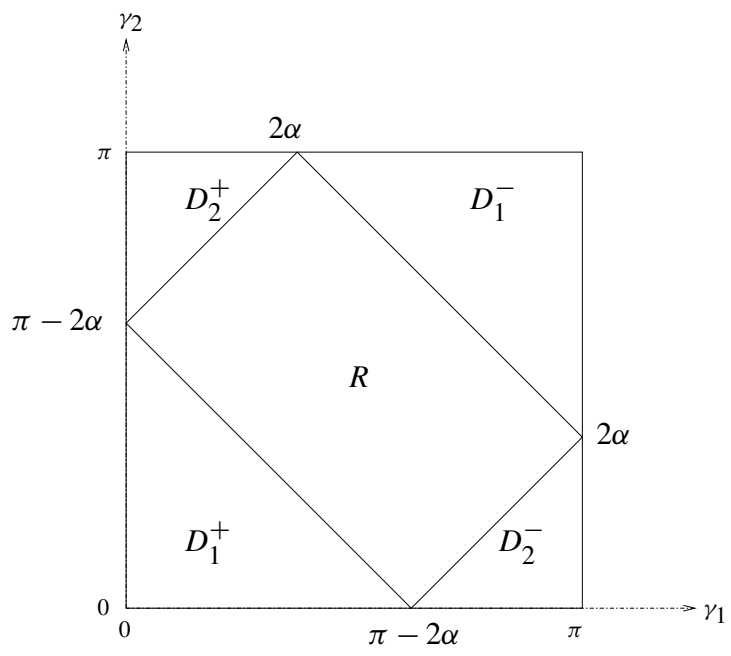

Figure 2. The Concus-Finn rectangle for convex corners.

of the behavior of a solution $f$ of (3)-(4) at the corner 0 ; here let $R, D_{1}^{ \pm}, D_{2}^{ \pm}$be the indicated open regions in the (open) square $(0, \pi) \times(0, \pi)$. If $\left(\gamma_{1}, \gamma_{2}\right)$ is in $\bar{R} \cap(0, \pi) \times(0, \pi)$, then $f$ is continuous at $\mathcal{O}$ [Concus and Finn 1996, Theorem 1; Lancaster and Siegel 1996b; 1996a, Corollary 4; Tam 1986]. If $\left(\gamma_{1}, \gamma_{2}\right) \in D_{1}^{ \pm}$, then $f$ is unbounded in any neighborhood of 0 and the capillary problem has no solution if $\kappa=0$ [Concus and Finn 1996; Finn 1996]. If $\left(\gamma_{1}, \gamma_{2}\right) \in D_{2}^{ \pm}$, then $f$ is bounded [Lancaster and Siegel 1996a, Proposition 1] but its continuity at $\mathcal{O}$ was unknown until recently. Concus and Finn discovered bounded solutions of (1)-(2) in domains with corners whose unit normals (i.e., Gauss maps) cannot extend continuously as functions of $x$ to a corner on the boundary of the domain [Finn 1988a, page 15; 1988b; 1996; Concus and Finn 1996, Example 2]. They formulated the conjecture that the solution $f$ of (1)-(2) must be discontinuous at 0 when $\left(\gamma_{1}, \gamma_{2}\right) \in D_{2}^{ \pm}$. Writing the conditions required for a pair of angles to be in $D_{2}^{ \pm}$yields the following formulation of their conjecture:

Concus-Finn conjecture. Suppose $0<\alpha<\pi / 2$, that the limits (5) exist and $0<$ $\gamma_{1}, \gamma_{2}<\pi$. If $2 \alpha+\left|\gamma_{1}-\gamma_{2}\right|>\pi$, then any solution of (3)-(4), with $H(x, z)=\kappa z+\lambda$, $\kappa \geq 0$, has a jump discontinuity at $O$.

This conjecture was proven for solutions of (3)-(4) (i.e., without the restriction that $H(x, z)=\kappa z+\lambda)$ in [Lancaster 2010]. ${ }^{1}$

Thus, when $2 \alpha \leq \pi,\left(\gamma_{1}, \gamma_{2}\right) \in D_{2}^{ \pm}$, and $f$ satisfies (3)-(4), $f$ is discontinuous at $\mathcal{O}$ and there is a countable set $\mathscr{I} \subset(-\alpha, \alpha)$ such that the radial limit function of $f$ at

\footnotetext{
${ }^{1}$ For convenience, we will abbreviate this reference as [L]. Similarly, [Lancaster and Siegel 1996a] and [Lancaster and Siegel 1996b] will be abbreviated [LS a] and [LS b], respectively.
} 


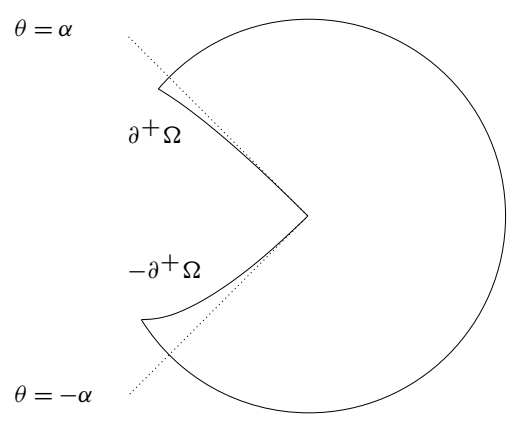

Figure 3. $\Omega$ with $2 \alpha>\pi$.

O, $R f$, defined by $R f(\alpha)=\lim _{\partial^{+} \Omega \ni x \rightarrow \mathcal{O}} f(x), R f(-\alpha)=\lim _{\partial^{-} \Omega \ni x \rightarrow \mathcal{O}} f(x)$ and

$$
R f(\theta)=\lim _{r \downarrow 0} f(r \cos \theta, r \sin \theta), \quad-\alpha<\theta<\alpha,
$$

is well-defined and continuous on $[-\alpha, \alpha] \backslash \mathscr{I}$ and behaves as in Proposition 1(i) of [LS b]; if $H(x, z)$ is strictly increasing in $z$ [LS a, §5] or real-analytic [LS b] for $x$ in a neighborhood of $\mathbb{O}$, then $\mathscr{I}=\varnothing$. (See [LS a], Step 3 of the proof of Theorem 1 and $\S 5$, and [LS b] regarding the sets $\mathscr{I}$ and cusp solutions.) We may assume for the moment that $\left(\gamma_{1}, \gamma_{2}\right) \in D_{2}^{+}$since the other case follows by interchanging $x_{1}$ and $x_{2}$; then Theorems 1 and 2 of [LS a] and Proposition 1 and Theorem 1 of [LS b] imply there is a countable set $\mathscr{I} \subset\left[\alpha_{1}, \alpha_{2}\right]$ such that

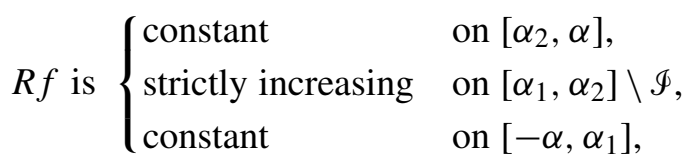

with $\alpha_{1}<\alpha_{2}, \alpha-\alpha_{2} \geq \gamma_{1}$ and $\alpha_{1}-(-\alpha) \geq \pi-\gamma_{2}$. In fact, determining the exact sizes of these radial limit fans when $f$ is discontinuous at 0 follows easily from [L]. (Notice that $D_{1}^{ \pm}=\varnothing$ if $2 \alpha=\pi$.)

Proposition 1.1. Let $\Omega$ be as above with $2 \alpha<\pi$ and $f$ be a bounded solution to (3)-(4). Suppose that $\left(\gamma_{1}, \gamma_{2}\right) \in D_{2}^{ \pm}$and that there exist constants $\underline{\gamma}^{ \pm}, \bar{\gamma}^{ \pm}$, $0<\underline{\gamma}^{ \pm} \leq \bar{\gamma}^{ \pm}<\pi$, satisfying

$$
\underline{\gamma}^{+}+\underline{\gamma}^{-}>\pi-2 \alpha \text { and } \bar{\gamma}^{+}+\bar{\gamma}^{-}<2 \alpha+\pi,
$$

so that $\underline{\gamma}^{ \pm} \leq \gamma^{ \pm}(s) \leq \bar{\gamma}^{ \pm}$for all $s, 0<s<s_{0}$, for some $s_{0}$. Then $R f(\theta)$ exists for $\theta \in[-\bar{\alpha}, \alpha] \backslash \mathscr{I}$ and $R f(\theta)$ is a continuous function of $\theta \in[-\alpha, \alpha] \backslash \mathscr{I}$, where $\mathscr{I}$ is a countable subset of $(-\alpha, \alpha)$. 
Case (I). If $\left(\gamma_{1}, \gamma_{2}\right) \in D_{2}^{+}$(i.e., $\left.\gamma_{1}-\gamma_{2}<2 \alpha-\pi\right)$ then $\alpha_{1}=-\alpha+\pi-\gamma_{2}, \alpha_{2}=\alpha-\gamma_{1}$ and

$$
R f \text { is } \begin{cases}\text { constant } & \text { on }\left[\alpha-\gamma_{1}, \alpha\right], \\ \text { strictly increasing } & \text { on }\left[-\alpha+\left(\pi-\gamma_{2}\right), \alpha-\gamma_{1}\right] \backslash \mathscr{I}, \\ \text { constant } & \text { on }\left[-\alpha,-\alpha+\left(\pi-\gamma_{2}\right)\right],\end{cases}
$$

where $\Phi$ is a countable subset of $\left[-\alpha+\left(\pi-\gamma_{2}\right), \alpha-\gamma_{1}\right]$.

Case (D). If $\left(\gamma_{1}, \gamma_{2}\right) \in D_{2}^{-}$(i.e., $\left.\gamma_{1}-\gamma_{2}>\pi-2 \alpha\right)$ then $\alpha_{1}=-\alpha+\gamma_{2}, \alpha_{2}=\alpha-\pi+\gamma_{1}$ and

$$
R f \text { is } \begin{cases}\text { constant } & \text { on }\left[\alpha-\left(\pi-\gamma_{1}\right), \alpha\right], \\ \text { strictly decreasing } & \text { on }\left[-\alpha+\gamma_{2}, \alpha-\left(\pi-\gamma_{1}\right)\right] \backslash \mathscr{I}, \\ \text { constant } & \text { on }\left[-\alpha,-\alpha+\gamma_{2}\right],\end{cases}
$$

where $\mathscr{I}$ is a countable subset of $\left[-\alpha+\gamma_{2}, \alpha-\pi+\gamma_{1}\right]$.

Proof. Using the information from [LS b] and [LS a] given above and assuming $\left(\gamma_{1}, \gamma_{2}\right) \in D_{2}^{+}$, we will argue by contradiction. Suppose that $\alpha_{2}<\alpha-\gamma_{1}$. Let

$$
\Omega_{0} \subset\left\{(r \cos \theta, r \sin \theta) \in \Omega: r>0, \alpha_{2}<\theta<\alpha-\gamma_{1} / 2\right\} .
$$

be an open set whose boundary $\partial \Omega_{0}$ contains $\left\{\theta=\alpha-\gamma_{1} / 2\right\}$ and is tangent to $\left\{\theta=\alpha_{2}\right\}$ at $O$ so that the appropriate analogue of $[\mathrm{L},(43)]$ tends to zero. Then $f$ is continuous on $\bar{\Omega}$ and, from Theorem 2.1 of [L], we obtain

(7) $\lim _{r \downarrow 0} \vec{n}_{f}\left(r \cos \left(\alpha-\frac{1}{2} \gamma_{1}\right), r \sin \left(\alpha-\frac{1}{2} \gamma_{1}\right)\right)=\left(-\sin \left(\alpha-\gamma_{1}\right), \cos \left(\alpha-\gamma_{1}\right), 0\right)$,

$$
\lim _{\substack{x \rightarrow 0 \\ x \in \partial \Omega_{0} \backslash\left\{\theta=\alpha-\frac{1}{2} \gamma_{1}\right\}}} \vec{n}_{f}(x)=\left(-\sin \alpha_{2}, \cos \alpha_{2}, 0\right) .
$$

Notice that the limiting contact angles at 0 are $\frac{1}{2} \gamma_{1}$ (on $\theta=\alpha-\frac{1}{2} \gamma_{1}$ ) and $\pi$ (on $\left.\theta=\alpha_{2}\right)$. Now, using Theorem 2.1 of [L], we see that the arguments in $\S 3$ of [L] yield a contradiction to the assumption that $\alpha_{2}<\alpha-\gamma_{1}$. (If $\gamma_{2}=\pi$ were allowed in Theorem 1.1 of [L], then a contradiction would follow immediately since $2 \alpha$ $=\alpha-\alpha_{2}-\frac{1}{2} \gamma_{1},\left|\gamma_{1}-\gamma_{2}\right|=\pi-\frac{1}{2} \gamma_{1}$ and $2 \alpha+\left|\gamma_{1}-\gamma_{2}\right|=\pi+\alpha-\gamma_{1}-\alpha_{2}>\pi$.) In the case that $\alpha_{1}>-\alpha+\pi-\gamma_{2}$ or $\left(\gamma_{1}, \gamma_{2}\right) \in D_{2}^{-}$, the proof follows in a similar manner.

The focus of this note is to give a direct proof of the nonconvex Concus-Finn conjecture and, when $\left(\gamma_{1}, \gamma_{1}\right) \in D_{1}^{ \pm} \cup D_{2}^{ \pm}$, establish the exact sizes of radial limit fans at reentrant corners and discuss the continuity of the Gauss map. We note that Danzhu Shi assumes the (convex) Concus-Finn conjecture holds when $\gamma_{1} \in\{0, \pi\}$ or $\gamma_{2} \in\{0, \pi\}$ and then, in her extremely interesting paper [Shi 2006], gives an argument for the proof of the nonconvex Concus-Finn conjecture. Unfortunately, 
these cases (e.g., $\gamma_{j} \in\{0, \pi\}, j=1,2$ ) are not covered in [L]. Our interest in proving the nonconvex Concus-Finn conjecture arises from our need, when determining the exact sizes of fans at reentrant corners, for the information developed during its proof (e.g., analogs of Theorem 2.1 of [L]) and from a belief in the value of presenting a proof which directly uses the ideas and techniques in [L].

\section{The nonconvex Concus-Finn conjecture}

The following theorem implies that the nonconvex Concus-Finn conjecture (cf. [Shi 2006]) is true; the proof will be given in Section 2B.

Theorem 2.1. Let $\Omega$ and $\gamma$ be as above with $\alpha \in\left[\frac{\pi}{2}, \pi\right]$. Let

$$
f \in C^{2}(\Omega) \cap C^{1, \rho}(B(\mathcal{O}, \delta) \cap \bar{\Omega} \backslash\{\mathcal{O}\})
$$

be a bounded solution of (3)-(4) with

$$
|H|_{\infty}=\sup _{\boldsymbol{x} \in \Omega}|H(\boldsymbol{x}, f(\boldsymbol{x}))|<\infty
$$

for some $\delta>0$ and $\rho \in(0,1)$. Suppose (5) holds and $\gamma_{1}, \gamma_{2} \in(0, \pi)$. Then $f$ is discontinuous at 0 whenever $\left|\gamma_{1}-\gamma_{2}\right|>2 \alpha-\pi$ or $\left|\gamma_{1}+\gamma_{2}-\pi\right|>2 \pi-2 \alpha$ (i.e., $\left.\left(\gamma_{1}, \gamma_{2}\right) \in D_{1}^{ \pm} \cup D_{2}^{ \pm}\right)$.

Throughout this section, we will consider $f$ to be a fixed solution of (3)-(4) that satisfies the hypotheses of this theorem. We may parametrize the graph of $f$ as in [LS a], using the unit disk $E=\left\{(u, v): u^{2}+v^{2}<1\right\}$ as our parameter domain. From Step 1 of the proof of Theorem 1 of [LS a] and $\S 3$ of [L], we see that there is a parametric description $X: \bar{E} \rightarrow \mathbb{R}^{3}$ of the closure $S$ of $S_{0}=\{(x, f(x)): x \in \Omega\}$,

$$
X(u, v)=(x(u, v), y(u, v), z(u, v)), \quad(u, v) \in \bar{E},
$$

such that:

(i) $X \in C^{2}\left(E: \mathbb{R}^{3}\right) \cap W^{1,2}\left(E: \mathbb{R}^{3}\right)$.

(ii) $X$ is a homeomorphism of $E$ onto $S_{0}$.

(iii) $X$ maps $\partial E$ onto $\{(x, f(x)): x \in \partial \Omega\} \cup\left(\{\mathcal{O}\} \times\left[z_{1}, z_{2}\right]\right)$, where

$$
z_{1}=\liminf _{\bar{\Omega} \ni x \rightarrow 0} f(x) \quad \text { and } \quad z_{2}=\limsup _{\bar{\Omega} \ni x \rightarrow 0} f(x) .
$$

(iv) $X$ is conformal on $E$ : $X_{u} \cdot X_{v}=0,\left|X_{u}\right|=\left|X_{v}\right|$ on $E$.

(v) Let $\tilde{H}(u, v)=H(X(u, v))$ denote the prescribed mean curvature of $\mathscr{S}_{f}$ at $X(u, v)$. Then $\triangle X:=X_{u u}+X_{v v}=\tilde{H} X_{u} \times X_{v}$.

(vi) $X \in C^{0}(\bar{E})$. 


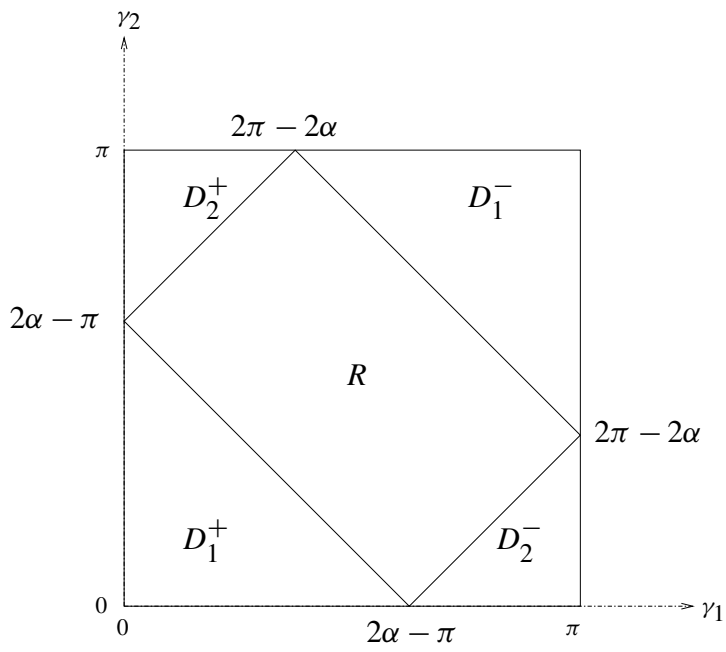

Figure 4. The Concus-Finn rectangle for nonconvex corners.

(vii) Writing $G(u, v)=(x(u, v), y(u, v)), G(\cos t, \sin t)$ moves clockwise about $\partial \Omega$ as $t$ increases, $0 \leq t \leq 2 \pi$, and $G$ is an orientation reversing homeomorphism from $E$ onto $\Omega$; $G$ maps $\bar{E}$ onto $\bar{\Omega}$ and, if $f$ is continuous at $\mathcal{O}$, then $G$ is a homeomorphism from $\bar{E}$ onto $\bar{\Omega}$.

(viii) Let $\pi_{S}: S^{2} \rightarrow \mathbb{C}$ denote the stereographic projection from the North Pole and define $g(u+i v)=\pi_{S}\left(\vec{n}_{f}(G(u, v))\right),(u, v) \in E$. Then

$$
\left|g_{\bar{\zeta}}\right|=\frac{1}{2}|\tilde{H}|\left(1+|g|^{2}\right)\left|X_{u}\right|,
$$

where $\zeta=u+i v, \partial / \partial \zeta=\frac{1}{2}(\partial / \partial u-i \partial / \partial v)$ and $\partial / \partial \bar{\zeta}=\frac{1}{2}(\partial / \partial u+i \partial / \partial v)$. For convenience when working with complex variables, set $E_{1}=\{\zeta \in \mathbb{C}:|\zeta|<1\}$.

(ix) The parametric Gauss map $N: E \rightarrow S^{2}$ is $N=\left(X_{u} \times X_{v}\right) /\left|X_{u} \times X_{v}\right|$ and satisfies $N(u, v)=\vec{n}_{f}(G(u, v)),(u, v) \in E$; the domain of $N$ is taken as the largest subset of $\bar{E}$ on which $N$ extends continuously.

It is convenient to introduce some notation. Suppose $V \subset \mathbb{R}^{2}$ with $\mathscr{O} \in \partial V$. For $t>0$, set $V_{t}=\left\{(x, y) \in V: x^{2}+y^{2}<t^{2}\right\}$. Let $s(V)$ denote the set of sequences in $V$ that converge to $\mathcal{O}$. If $h \in C^{1}(V)$, we define $\Pi_{h}(V)=\bigcap_{t>0} \overline{\vec{n}_{h}\left(V_{t}\right)}$; then

$$
\Pi_{h}(V)=\left\{Y \in S^{2}: \text { there exists }\left(x_{j}\right) \in s(V) \text { such that } Y=\lim _{j \rightarrow \infty} \vec{n}_{h}\left(x_{j}\right)\right\} .
$$

Without assuming that $f$ is or is not continuous at $\mathbb{O}$, we have:

Lemma 2.2. Let $\Lambda$ be an open, connected, simply connected subset of $\Omega$ with $\mathrm{O}$ in $\partial \Lambda$ and suppose that there is a rotation $M$ of $\mathbb{R}^{2}$ about 0 such that

$$
\left\{\left(M\left(y_{1}, y_{2}\right), y_{3}\right): Y \in \Pi_{f}(\Lambda)\right\}
$$


is contained in a compact subset of $\left\{Y \in S^{2}: y_{2}>0, y_{3} \leq 0\right\}$. Let $\phi$ be a conformal map from $E$ to $G^{-1}(\Lambda)$ and define

$$
\tilde{g}(u+i v)=\pi_{S}\left(\vec{n}_{f}(G \circ \phi(u, v))\right), \quad(u, v) \in E .
$$

Then there exists $p>2$ such that

$$
\tilde{g}(\zeta)=\psi(\zeta)+h(\zeta), \quad \zeta \in E_{1},
$$

where $\psi$ is a holomorphic function on $E_{1}$ and $h \in L^{\infty}\left(E_{1}\right)$ is a Hölder continuous function on $\bar{E}_{1}$ with Hölder exponent $\mu=(p-2) / p$.

Proof. In $\S 3$ of [L], the fact that the limits at 0 of the Gauss map are contained in a compact subset of $\left\{Y \in S^{2}: y_{2}>0, y_{3} \leq 0\right\}$ implies that $(u, v) \mapsto(z(u, v), x(u, v))$ is quasiconformal and has a quasiconformal extension to $\mathbb{R}^{2}$; Gehring's lemma and the isothermal parametrization imply $X \in W^{1, p}$ for some $p>2$ and the classical literature implies $g=\psi+h$ with $\psi$ and $h$ as above. We can argue as in $\S 3$ of [L]; we find that $X \in W^{1, p}\left(E: \mathbb{R}^{3}\right)$ for some $p>2$ and

$$
\tilde{g}(\zeta)=\psi(\zeta)+h(\zeta)
$$

where $\psi$ is a holomorphic function and $h \in L^{\infty}\left(E_{1}\right)$ is an uniformly Hölder continuous function on $E_{1}$ with Hölder exponent $\mu$.

Remark 2.3. Notice that $\tilde{g}=g \circ \phi_{1}$, where $\phi_{1}$ is a conformal map from $E_{1}$ onto $\left\{u+i v:(u, v) \in G^{-1}(\Lambda)\right\}$.

2A. Image of the Gauss map. The (nonparametric) Gauss map on $\mathscr{S}_{f}$ is the (downward) unit normal map to $\mathscr{Y}_{f}$ when this is defined and equals $\vec{n}_{f}$ on $\mathscr{S}_{f} \cap(\Omega \times \mathbb{R})$; here we consider $\vec{n}_{f}: \Omega \times \mathbb{R} \rightarrow S_{-}^{2}$ by letting $(x, t) \mapsto \vec{n}_{f}(x)$. In this section, we characterize in Theorems 2.4 and 2.5 the behavior of the limits at points of $\{0\} \times \mathbb{R}$ of the Gauss map for the graph of $f$ when $\left(\gamma_{1}, \gamma_{2}\right) \notin \bar{R}$. Let $S_{-}^{2}=\left\{\omega \in \mathbb{R}^{3}:|\omega|=1, \omega_{3} \leq 0\right\}$ be the (closed) lower half of the unit sphere.

Theorem 2.4. Let $2 \alpha>\pi$ and $\Omega$ and $\gamma$ be as in Section 1 and suppose (5) holds with $\gamma_{1}, \gamma_{2} \in(0, \pi)$. Let $\beta \in(-\alpha, \alpha)$ and $\left(x_{j}\right) \in s(\Omega)$ such that

$$
\lim _{j \rightarrow \infty} \frac{x_{j}}{\left|x_{j}\right|}=(\cos \beta, \sin \beta) \text {. }
$$

Let us write $\omega(\theta)=(\cos \theta, \sin \theta, 0)$ for $\theta \in \mathbb{R}$.

$\left(D_{2}^{+}\right)$If $\left(\gamma_{1}, \gamma_{2}\right) \in D_{2}^{+}\left(\right.$i.e., $\left.\gamma_{1}-\gamma_{2}<\pi-2 \alpha\right)$ then

$$
\lim _{j \rightarrow \infty} \vec{n}_{f}\left(x_{j}\right)= \begin{cases}\omega\left(\alpha-\gamma_{1}+\frac{\pi}{2}\right) & \text { if } \beta \in\left[\alpha-\gamma_{1}, \alpha\right), \\ \omega\left(\beta+\frac{\pi}{2}\right) & \text { if } \beta \in\left[-\alpha+\left(\pi-\gamma_{2}\right), \alpha-\gamma_{1}\right], \\ \omega\left(-\alpha-\gamma_{2}+\frac{3 \pi}{2}\right) & \text { if } \beta \in\left(-\alpha,-\alpha+\left(\pi-\gamma_{2}\right)\right] .\end{cases}
$$


$\left(D_{2}^{-}\right)$If $\left(\gamma_{1}, \gamma_{2}\right) \in D_{2}^{-}\left(\right.$i.e., $\left.\gamma_{1}-\gamma_{2}>2 \alpha-\pi\right)$ then

$$
\lim _{j \rightarrow \infty} \vec{n}_{f}\left(x_{j}\right)= \begin{cases}\omega\left(\alpha+\gamma_{1}-\frac{3 \pi}{2}\right) & \text { if } \beta \in\left[\alpha+\gamma_{1}-\pi, \alpha\right), \\ \omega\left(\beta-\frac{\pi}{2}\right) & \text { if } \beta \in\left[-\alpha+\gamma_{2}, \alpha+\gamma_{1}-\pi\right], \\ \omega\left(-\alpha+\gamma_{2}-\frac{\pi}{2}\right) & \text { if } \beta \in\left(-\alpha,-\alpha+\gamma_{2}\right] .\end{cases}
$$

Proof. Let us assume $\left(\gamma_{1}, \gamma_{2}\right) \in D_{2}^{ \pm}$. Let $\beta \in(-\alpha, \alpha)$ and $\left(x_{j}\right)$ be an arbitrary sequence in $\Omega$ converging to 0 and satisfying (13). Since $\left(\vec{n}_{f}\left(x_{j}\right): j \in \mathbb{N}\right)$ is a sequence in the compact set $S_{-}^{2}$, there is a subsequence of $\left(x_{j}: j \in \mathbb{N}\right)$, still denoted $\left(x_{j}: j \in \mathbb{N}\right)$, and $\theta \in\left(-\frac{\pi}{2}, \frac{3 \pi}{2}\right], \tau \in[0,1]$ such that $\left(\vec{n}_{f}\left(x_{j_{k}}\right): k \in \mathbb{N}\right)$ is convergent and

$$
\lim _{k \rightarrow \infty} \vec{n}_{f}\left(x_{j_{k}}\right)=\left(\tau \cos \theta, \tau \sin \theta,-\sqrt{1-\tau^{2}}\right) .
$$

Using [Jeffres and Lancaster 2008] and the techniques and arguments in $\S 2$ of [L], we see that $\tau=1, \lim _{k \rightarrow \infty} \vec{n}_{f}\left(x_{j_{k}}\right)=\omega(\theta)$, and $\omega(\theta)$ is normal to $\partial \mathscr{P}$ and points into $\mathscr{P}$, where $\omega(\beta) \in \partial \mathscr{P}$ and $\mathscr{P}$ is given in Theorem 2.2 of [Jeffres and Lancaster 2008]. (In $\S 2$ of [L], the function $u(x)=f(x)-R f(\beta)$ is blown up about $(0,0,0)$; that is, the graphs of a subsequence of the sequence $\left(u_{j}\right)$ in $C^{2}(\Omega)$, where $u_{j}$ is defined by $u_{j}(x)=\left(f\left(\epsilon_{j} x\right)-R f(\beta)\right) / \epsilon_{j}$ and $\epsilon_{j}=\left|x_{j}\right|$ for $j \in \mathbb{N}$, are shown to converge to the intersection of $\Omega \times \mathbb{R}$ with a vertical plane $\pi_{1}$. The (downward) unit normal to $\pi_{1}$ is shown to be normal to the vertical plane $\partial \mathscr{P}$ which contains $(\cos \beta, \sin \beta, 0)$ and point into $\mathscr{P}$, where $\mathscr{P}$ satisfies Theorem 2.1 of [Jeffres and Lancaster 2007].)

If $\left(\gamma_{1}, \gamma_{2}\right) \in D_{2}^{+}$, then the conclusions of Theorem 2.4 follow from Corollary 2.4 of [Jeffres and Lancaster 2008]; Figure 5 illustrates the graph of the argument of $\vec{n}(\beta)=\lim _{r \downarrow 0} \vec{n}_{f}(r \cos \beta, r \sin \beta)$. If $\left(\gamma_{1}, \gamma_{2}\right) \in D_{2}^{-}$, then the conclusions of Theorem 2.4 follow from Corollary 2.5 of [ibid.].

Suppose that $\alpha \in\left(\frac{\pi}{2}, \pi\right], \gamma_{1}, \gamma_{2} \in(0, \pi)$ and $\gamma_{1}+\gamma_{2}<2 \alpha-\pi$. Let us define $\mathscr{F}=\mathscr{F}\left(\alpha, \gamma_{1}, \gamma_{2}\right)$ as follows: Set

$$
\begin{gathered}
\mathscr{F}_{1}=\left[-\alpha,-\alpha-\gamma_{2}+\pi\right] \times\left\{-\alpha-\gamma_{2}-\pi / 2\right\}, \\
\mathscr{F}_{2}=\left[-\alpha,-\alpha+\gamma_{2}+\pi\right] \times\left\{-\alpha+\gamma_{2}-\pi / 2\right\}, \\
\mathscr{F}_{3}=\left[\alpha-\gamma_{1}-\pi, \alpha\right] \times\left\{\alpha-\gamma_{1}-3 \pi / 2\right\}, \\
\mathscr{F}_{4}=\left[\alpha+\gamma_{1}-\pi, \alpha\right] \times\left\{\alpha+\gamma_{1}-3 \pi / 2\right\}, \\
\mathscr{F}_{5}=\left\{(\beta, \beta-\pi / 2): \beta \in\left[-\alpha+\gamma_{2}, \alpha+\gamma_{1}-\pi\right]\right\}, \\
\mathscr{F}_{6}=\left\{(\beta, \beta-3 \pi / 2): \beta \in\left[-\alpha+\gamma_{2}+\pi, \alpha-\gamma_{1}\right]\right\}, \\
\mathscr{F}_{7}=\left\{\left(\beta+t,-\alpha+\gamma_{2}-\pi / 2+t\right): \begin{array}{c}
\beta \in\left[-\alpha+\gamma_{2},-\alpha+\gamma_{2}+\pi\right] \\
t \in\left[0,2 \alpha-\pi-\gamma_{1}-\gamma_{2}\right]
\end{array}\right\},
\end{gathered}
$$

and define $\mathscr{F}=\bigcup_{j=1}^{7} \mathscr{F}_{j}=\mathscr{F}_{1} \cup \ldots \cup \mathscr{F}_{7}$ (see Figures 6,7 and 8 for illustrations). 
378

KIRK E. LANCASTER

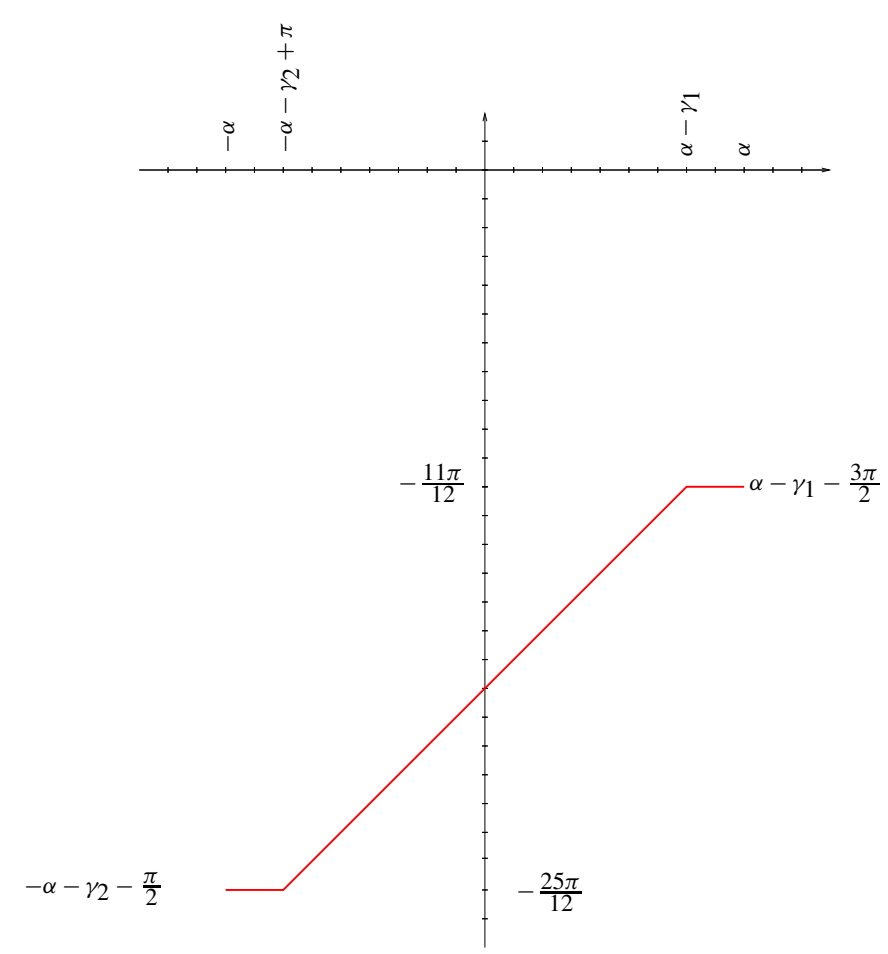

Figure 5. $\left\{\left(\beta, \arg \left(\vec{m}_{f}(\beta)\right)\right)\right\}$ at a $D_{2}^{+} \operatorname{corner} ; \alpha=\frac{3 \pi}{4}, \gamma_{1}=\frac{\pi}{6}, \gamma_{2}=\frac{5 \pi}{6}$.

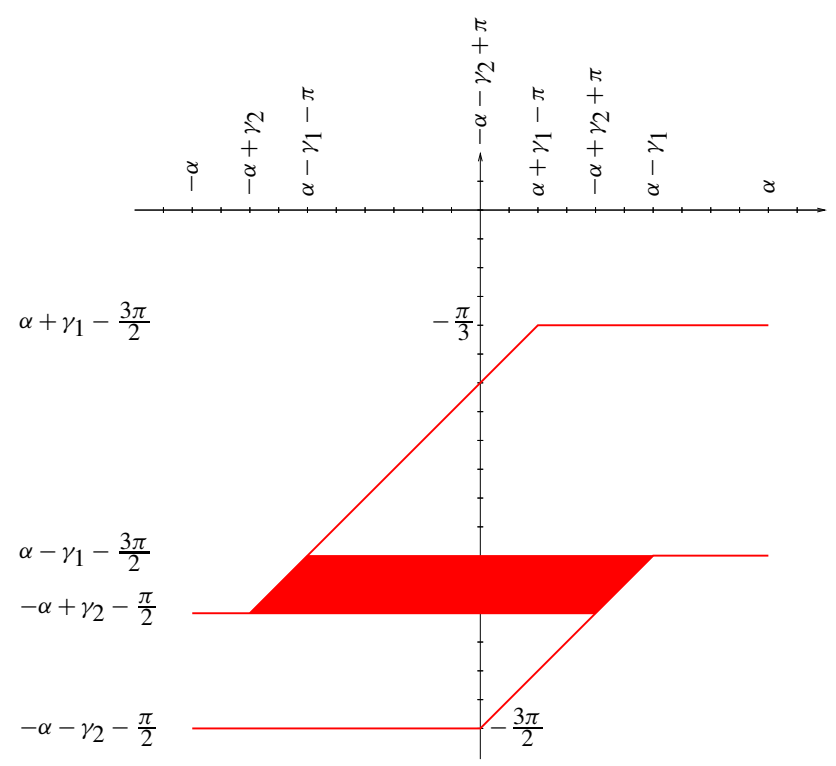

Figure 6. $\mathscr{F}$ at a $D_{1}^{+}$corner; $\alpha=\frac{5 \pi}{6}, \gamma_{1}=\frac{\pi}{3}, \gamma_{2}=\frac{\pi}{6}$. 


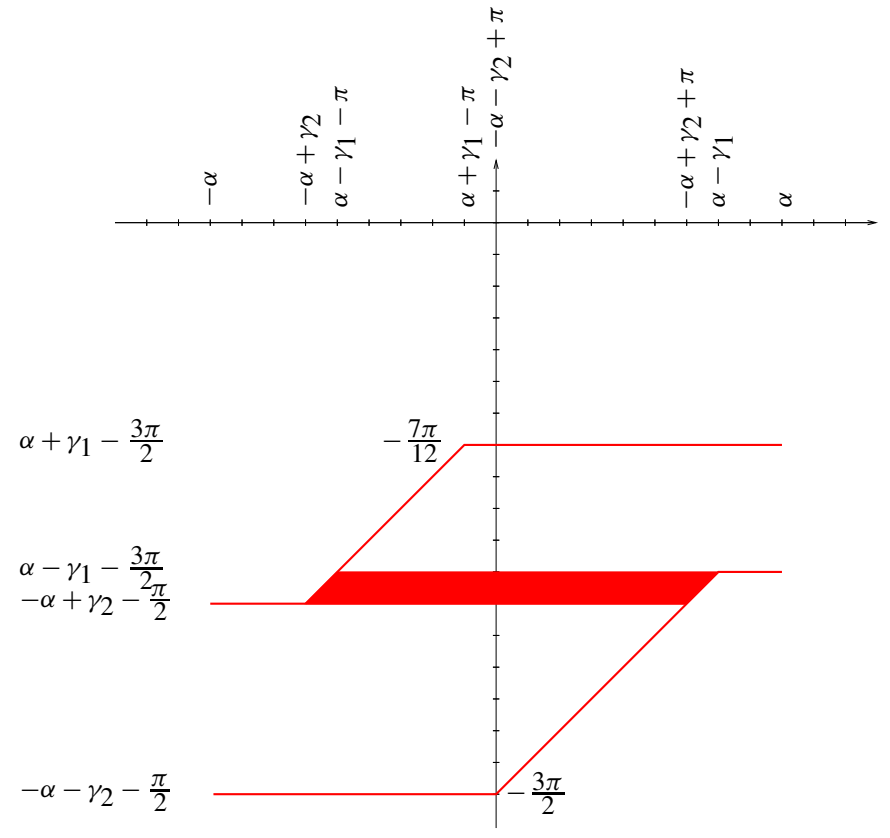

Figure 7. $\mathscr{F}$ at a $D_{1}^{+}$corner; $\alpha=\frac{3 \pi}{4}, \gamma_{1}=\frac{\pi}{6}, \gamma_{2}=\frac{\pi}{4}$.

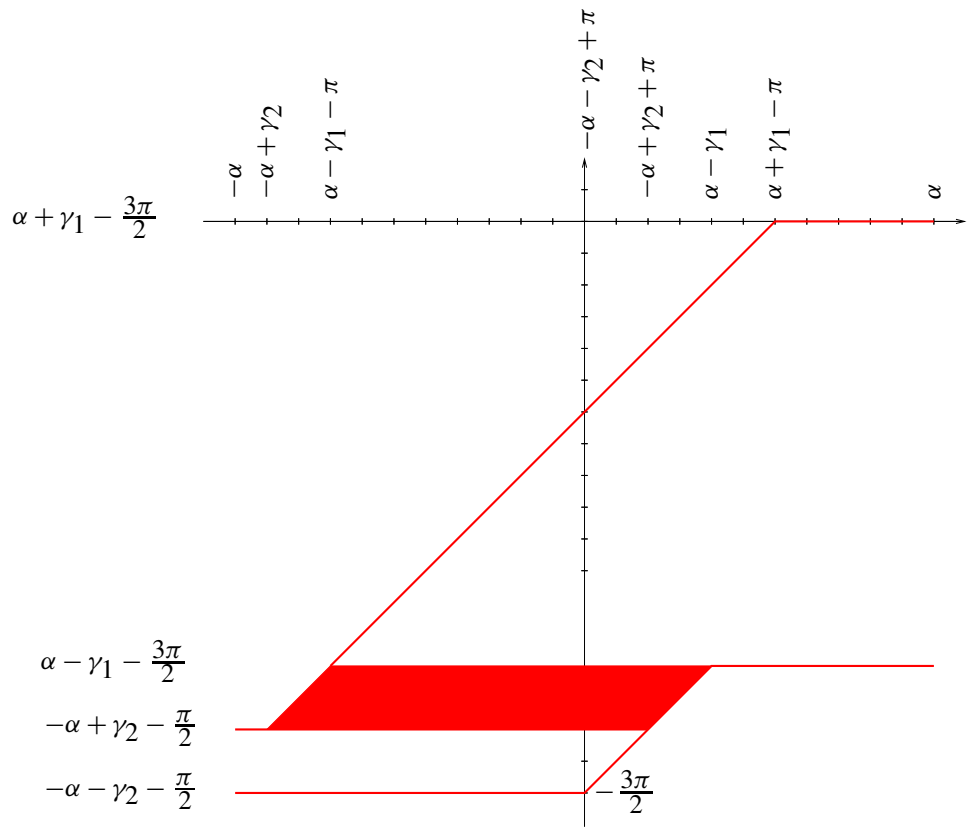

Figure 8. $\mathscr{F}$ at a $D_{1}^{+} \operatorname{corner} ; \alpha=\frac{11 \pi}{12}, \gamma_{1}=\frac{7 \pi}{12}, \gamma_{2}=\frac{\pi}{12}$. 
Theorem 2.5. Let $2 \alpha>\pi$ and $\Omega$ and $\gamma$ be as in Section 1 and suppose (5) holds with $\gamma_{1}, \gamma_{2} \in(0, \pi)$. Let $\beta \in(-\alpha, \alpha)$ and $\left(x_{j}\right) \in s(V)$ such that

$$
\lim _{j \rightarrow \infty} \frac{x_{j}}{\left|x_{j}\right|}=(\cos \beta, \sin \beta) .
$$

Continuing to write $\omega(\theta)=(\cos \theta, \sin \theta, 0)$ for $\theta \in \mathbb{R}$, we see:

(i) Suppose $\left(\gamma_{1}, \gamma_{2}\right) \in D_{1}^{+}$(i.e., $\left.\gamma_{1}+\gamma_{2}<2 \alpha-\pi\right), \lim _{j \rightarrow \infty} \vec{n}_{f}\left(x_{j}\right)$ exists and

$$
\lim _{j \rightarrow \infty} \vec{n}_{f}\left(x_{j}\right)=\omega(\theta) \quad \text { for some } \theta \in \mathbb{R} .
$$

Then $(\beta, \theta) \in \mathscr{F}$.

(ii) Suppose $\left(\gamma_{1}, \gamma_{2}\right) \in D_{1}^{-}$(i.e., $\left.\gamma_{1}+\gamma_{2}>2 \alpha+\pi\right), \lim _{j \rightarrow \infty} \vec{n}_{f}\left(x_{j}\right)$ exists and

$$
\lim _{j \rightarrow \infty} \vec{n}_{f}\left(x_{j}\right)=\omega(\theta) \quad \text { for some } \theta \in \mathbb{R} .
$$

Then $(-\beta, \theta) \in \mathscr{F}_{\text {. }}$

(iii) Connectedness at $\beta$ : Suppose $\left(\gamma_{1}, \gamma_{2}\right) \in D_{1}^{+}$and $\left(x_{j}\right),\left(y_{j}\right) \in s(\Omega)$ such that

$$
\begin{gathered}
\lim _{j \rightarrow \infty} \frac{x_{j}}{\left|x_{j}\right|}=\lim _{j \rightarrow \infty} \frac{y_{j}}{\left|y_{j}\right|}=(\cos \beta, \sin \beta), \\
\lim _{j \rightarrow \infty} \vec{n}_{f}\left(x_{j}\right)=\omega\left(\theta_{1}\right) \text { and } \lim _{j \rightarrow \infty} \vec{n}_{f}\left(x_{j}\right)=\omega\left(\theta_{2}\right),
\end{gathered}
$$

for some $\theta_{1} \leq \theta_{2}$ such that $\left(\beta, \theta_{1}\right),\left(\beta, \theta_{2}\right) \in \mathscr{F}$. Then the set $\left\{\theta \in\left[\theta_{1}, \theta_{2}\right]:(\beta, \theta) \in \mathscr{F}\right\}$ must be connected.

(iv) Connectedness: Suppose $\left(\gamma_{1}, \gamma_{2}\right) \in D_{1}^{+}$. Let $\beta_{1}, \beta_{2} \in(-\alpha, \alpha)$ with $\beta_{1} \leq \beta_{2}$. Suppose $\left(x_{j}\right),\left(y_{j}\right) \in s(\Omega)$ such that

$$
\begin{gathered}
\lim _{j \rightarrow \infty} \frac{x_{j}}{\left|x_{j}\right|}=\left(\cos \beta_{1}, \sin \beta_{1}\right), \quad \lim _{j \rightarrow \infty} \frac{y_{j}}{\left|y_{j}\right|}=\left(\cos \beta_{2}, \sin \beta_{2}\right), \\
\lim _{j \rightarrow \infty} \vec{n}_{f}\left(x_{j}\right)=\omega\left(\theta_{1}\right) \quad \text { and } \quad \lim _{j \rightarrow \infty} \vec{n}_{f}\left(x_{j}\right)=\omega\left(\theta_{2}\right),
\end{gathered}
$$

for some $\theta_{1}, \theta_{2}$ such that $\left(\beta_{1}, \theta_{1}\right),\left(\beta_{2}, \theta_{2}\right) \in \mathscr{F}$. Set $L=\left[\min \left\{\theta_{1}, \theta_{2}\right\}, \max \left\{\theta_{1}, \theta_{2}\right\}\right]$. Then the set $\mathscr{F} \cap\left(\left[\beta_{1}, \beta_{2}\right] \times L\right)$ must be connected.

Proof. The proof of Theorem 2.5 (i) and (ii) is essentially the same as that of Theorem 2.4 with Corollaries 2.6 and 2.7 of [Jeffres and Lancaster 2008] replacing Corollaries 2.4 and 2.5 respectively. Conclusion (iii) follows from (i) by standard arguments (e.g., proof of Lemma 4.2). Conclusion (iv) follows from (i) by standard arguments which take into account the specific geometry of $\mathscr{F}_{\text {. }}$. 
2B. Proof of Theorem 2.1. Assume $\left(\gamma_{1}, \gamma_{2}\right) \in D_{1}^{ \pm} \cup D_{2}^{ \pm}, f$ satisfies (3) in $\Omega$ and (4) on $B\left(\mathcal{O}, \delta^{*}\right) \cap \partial \Omega \backslash\{\mathcal{O}\}$ and $f$ is continuous at $\mathcal{O}$; then $f$ is bounded in a neighborhood of $\mathrm{O}$. Since $f$ is continuous at $\mathrm{O}$, we have the following modifications of (i)-(viii) in Section 2A:

(iii) $^{\prime} X$ maps $\partial E$ strictly monotonically onto $\{(x, f(x)): x \in \partial \Omega\}$.

(vi) $X \in C^{0}(\bar{E})$ and $X(1,0)=\left(0,0, z_{0}\right)$, where $z_{0}=f(0,0)$.

(vii)' Continuing to write $G(u, v)=(x(u, v), y(u, v)), G(\cos t, \sin t)$ moves clockwise about $\partial \Omega$ as $t$ increases, $0 \leq t \leq 2 \pi$, and $G$ is an orientation reversing homeomorphism from $\bar{E}$ onto $\bar{\Omega}$.

We will prove Theorem 2.1 in the cases $\left(\gamma_{1}, \gamma_{2}\right) \in D_{2}^{+}$and $\left(\gamma_{1}, \gamma_{2}\right) \in D_{1}^{+}$; this will suffice to prove the lemma since the mapping

$$
\mathbb{R}^{3} \rightarrow \mathbb{R}^{3}, \quad\left(x_{1}, x_{2}, x_{3}\right) \mapsto\left(x_{1},-x_{2},-x_{3}\right),
$$

converts a $D_{2}^{-}$corner into a $D_{2}^{+}$corner and converts a $D_{1}^{-}$corner into a $D_{1}^{+}$corner.

Suppose $\left(\gamma_{1}, \gamma_{2}\right) \in D_{2}^{+}$. Set $\theta_{1}=\left(\pi-\left(\gamma_{1}+\gamma_{2}\right)\right) / 2$ and let $\theta_{2} \in\left(\alpha-\gamma_{1}, \alpha\right)$. By choosing $\delta_{0}>0$ small, we may assume

$$
\Omega^{*}=\left\{(r \cos \theta, r \sin \theta): 0<r<\delta_{0}, \theta_{1}<\theta<\theta_{2}\right\} \subset \Omega .
$$

Notice that Theorem $2.4\left(D_{2}^{+}\right)$implies

$$
\Pi_{f}\left(\Omega^{*}\right)=\left\{(\cos \theta, \sin \theta, 0): \theta_{1}+\frac{1}{2} \pi \leq \theta \leq \alpha-\gamma_{1}+\frac{1}{2} \pi\right\} .
$$

Since $\alpha-\gamma_{1}-\theta_{1}=\frac{1}{2}\left(2 \alpha-\pi-\gamma_{1}+\gamma_{2}\right) \in(2 \alpha-\pi, \alpha) \subset(0, \pi)$, the hypotheses of Lemma 2.2 are satisfied (with $M$ a rotation through an angle of $\pi / 2-\alpha$ ). If $\phi$ is a conformal map from $E$ onto $G^{-1}\left(\Omega^{*}\right)$ which maps $(1,0)$ to $(1,0)$ and $\tilde{g}$ is defined by (10), then Lemma 2.2 implies there exists $p>2$ such that

$$
\tilde{g}(\zeta)=\psi(\zeta)+h(\zeta)
$$

where $\psi$ is a holomorphic function and $h \in L^{\infty}\left(E_{1}\right)$ is a Hölder continuous function on $\bar{E}_{1}$ with Hölder exponent $\mu=(p-2) / p$. The assumption that $f$ is continuous at $\mathcal{O}$ yields a contradiction as in $\S 3$ of [L] (i.e., the Phragmén-Lindelöf theorem is violated).

Now suppose $\left(\gamma_{1}, \gamma_{2}\right) \in D_{1}^{+}$. Let $\theta_{1} \in\left(-\alpha,-\alpha+\gamma_{2}\right)$ and $\theta_{2} \in\left(\alpha-\gamma_{1}, \alpha\right)$ and choose $\delta_{0}>0$ small enough that

$$
\Omega^{*}=\left\{(r \cos \theta, r \sin \theta): 0<r<\delta_{0}, \theta_{1}<\theta<\theta_{2}\right\} \subset \Omega .
$$

Using Theorem 2.5, we see that

$$
\Pi_{f}\left(\Omega^{*}\right) \subset\left\{(\cos \theta, \sin \theta, 0): \beta \in(-\alpha, \alpha),(\beta, \theta) \in \mathscr{F}_{L}\right\},
$$




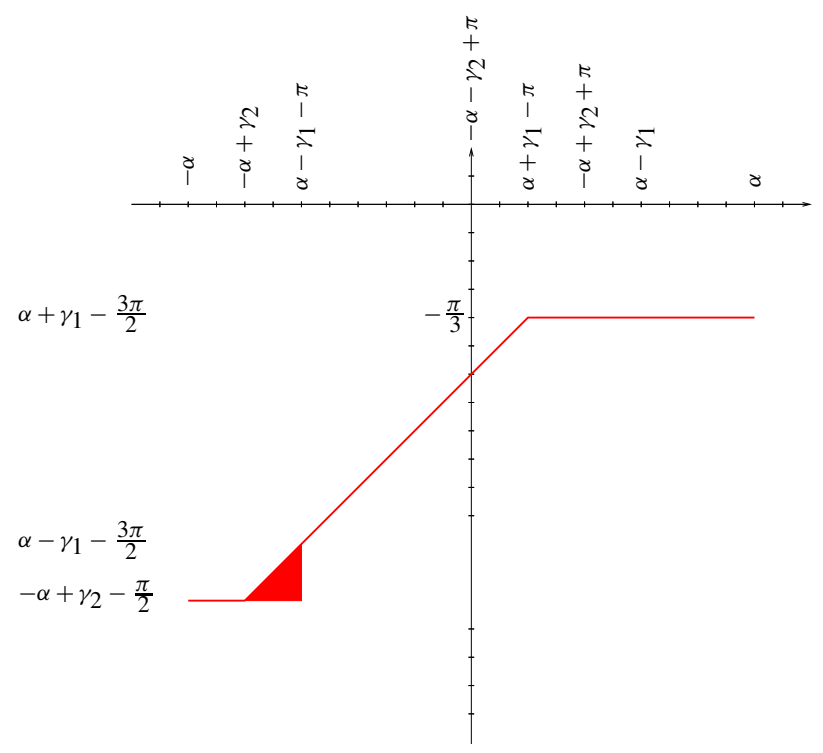

Figure 9. $\mathscr{F}_{A}$ at a $D_{1}^{+}$corner; $\alpha=\frac{5 \pi}{6}, \gamma_{1}=\frac{\pi}{3}, \gamma_{2}=\frac{\pi}{6}$.

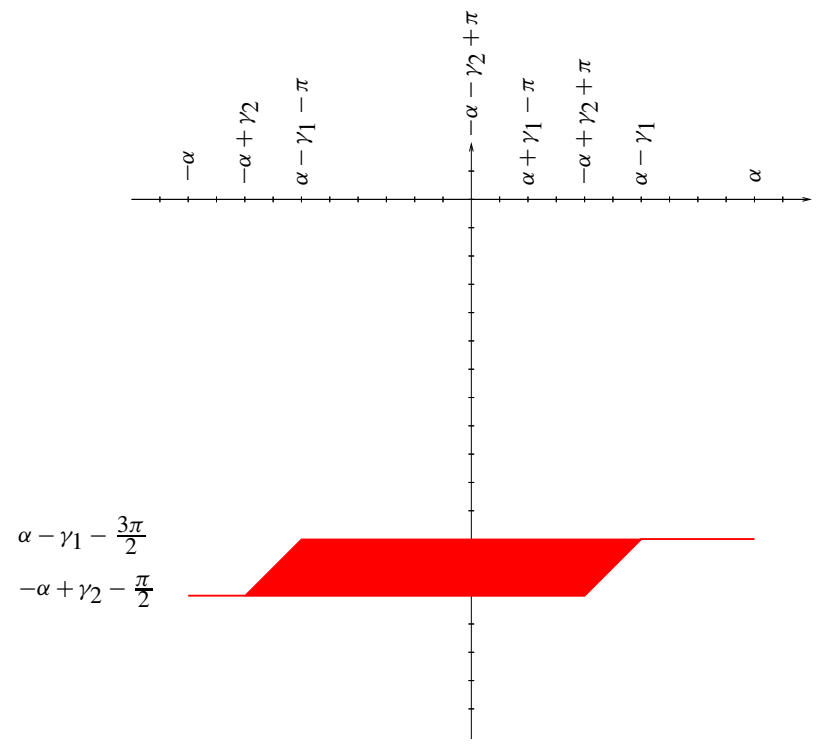

Figure 10. $\mathscr{F}_{B}$ at a $D_{1}^{+} \operatorname{corner} ; \alpha=\frac{5 \pi}{6}, \gamma_{1}=\frac{\pi}{3}, \gamma_{2}=\frac{\pi}{6}$.

where $\mathscr{F}_{L}$ is one of the sets $\mathscr{F}_{A}, \mathscr{F}_{B}$ or $\mathscr{F}_{C}$ illustrated in Figures 9, 10 and 11 respectively. When $\mathscr{F}_{L}$ is $\mathscr{F}_{A}$ or $\mathscr{F}_{C}$, the proof is essentially that same as that above for the case in which $\left(\gamma_{1}, \gamma_{2}\right) \in D_{2}^{+}$. When $\mathscr{F}_{L}$ is $\mathscr{F}_{B}$, the proof is essentially that same as that in $\S 3$ of $[\mathrm{L}]$. 


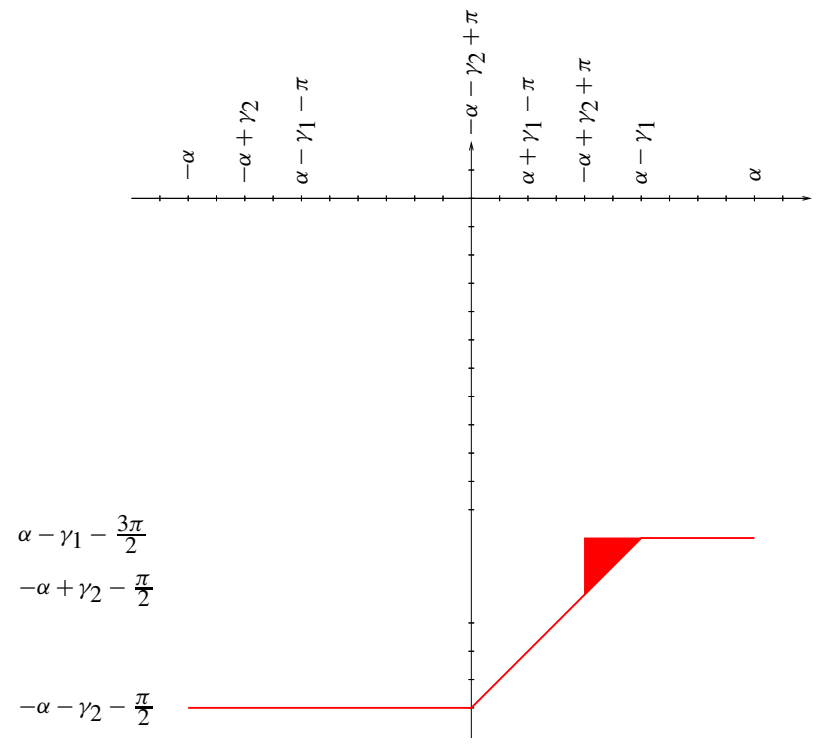

Figure 11. $\mathscr{F}_{C}$ at a $D_{1}^{+}$corner; $\alpha=\frac{5 \pi}{6}, \gamma_{1}=\frac{\pi}{3}, \gamma_{2}=\frac{\pi}{6}$.

\section{The exact sizes of fans}

We recall that a solution $f \in C^{2}(\Omega) \cap C^{1, \rho}(B(\mathcal{O}, \delta) \cap \bar{\Omega} \backslash\{\mathcal{O}\})$ of (3)-(4) is unbounded if $\alpha<\pi / 2$ and $\left(\gamma_{1}, \gamma_{2}\right) \in D_{1}^{ \pm}$, for some $\delta>0$ and $\rho \in(0,1)$. The following lemma justifies the definition of $\vec{m}_{f}:(-\alpha, \alpha) \rightarrow S_{-}^{2}$, given by

$$
\vec{m}_{f}(\beta)=\lim _{j \rightarrow \infty} \vec{n}_{f}\left(x_{j}\right) \text { whenever }\left(x_{j}\right) \in s(\Omega) \text { with } \lim _{j \rightarrow \infty} \frac{x_{j}}{\left|x_{j}\right|}=(\cos \beta, \sin \beta),
$$

when $\left(\gamma_{1}, \gamma_{2}\right) \in D_{1}^{ \pm} \cup D_{2}^{ \pm}$.

Lemma 3.1. Let $\Omega$ and $\gamma$ be as in Section 1 , with $\alpha \in[0, \pi]$. For some $\rho \in(0,1)$ and $\delta>0$, suppose $f \in C^{2}(\Omega) \cap C^{1, \rho}(B(\mathcal{O}, \delta) \cap \bar{\Omega} \backslash\{\mathcal{O}\})$ is a bounded solution of (3)-(4) with $|H|_{\infty}=\sup _{\boldsymbol{x} \in \Omega}|H(\boldsymbol{x}, f(\boldsymbol{x}))|<\infty$. Suppose (5) holds, $\gamma_{1}, \gamma_{2} \in(0, \pi)$ and $\left(\gamma_{1}, \gamma_{2}\right) \in D_{1}^{ \pm} \cup D_{2}^{ \pm}$; that is, either $\alpha \in\left[0, \frac{\pi}{2}\right)$ and $\left|\gamma_{1}-\gamma_{2}\right|>\pi-2 \alpha$ holds or $\alpha \in\left[\frac{\pi}{2}, \pi\right]$ and one of $\left|\gamma_{1}-\gamma_{2}\right|>2 \alpha-\pi$ or $\left|\gamma_{1}+\gamma_{2}-\pi\right|>2 \pi-2 \alpha$ holds. Then the Gauss map from $\mathscr{Y}_{f}$ to $S_{-}^{2}$ is continuous on $\mathscr{Y}_{f} \cap(\overline{\Omega(\epsilon)} \times \mathbb{R})$ for each $\epsilon>0$, where $\Omega(\epsilon)=\{(r \cos \theta, r \sin \theta) \in \Omega: r>0,|\theta|<\alpha-\epsilon\}$. In particular, $\vec{m}_{f}(\beta)$ exists for all $\beta \in(-\alpha, \alpha)$ and $\vec{m}_{f} \in C^{0}\left((-\alpha, \alpha): S_{-}^{2}\right)$.

Proof. Using Theorem 2.1 of [LS a] when $\alpha<\pi / 2$ and $\left(\gamma_{1}, \gamma_{2}\right) \in D_{2}^{ \pm}$and Theorems 2.4 and 2.5 and the proof of Theorem 2.1 when $\alpha \geq \pi / 2$ and $\left(\gamma_{1}, \gamma_{2}\right)$ is in $D_{1}^{ \pm} \cup D_{2}^{ \pm}$, we see that the hypotheses of Lemma 2.2 are satisfied when $\epsilon>0$ and $\Lambda=\Omega(\epsilon)$. Therefore the restriction of the Gauss map to $\mathscr{Y}_{f} \cap(\overline{\Omega(\epsilon)} \times \mathbb{R})$ is continuous. 


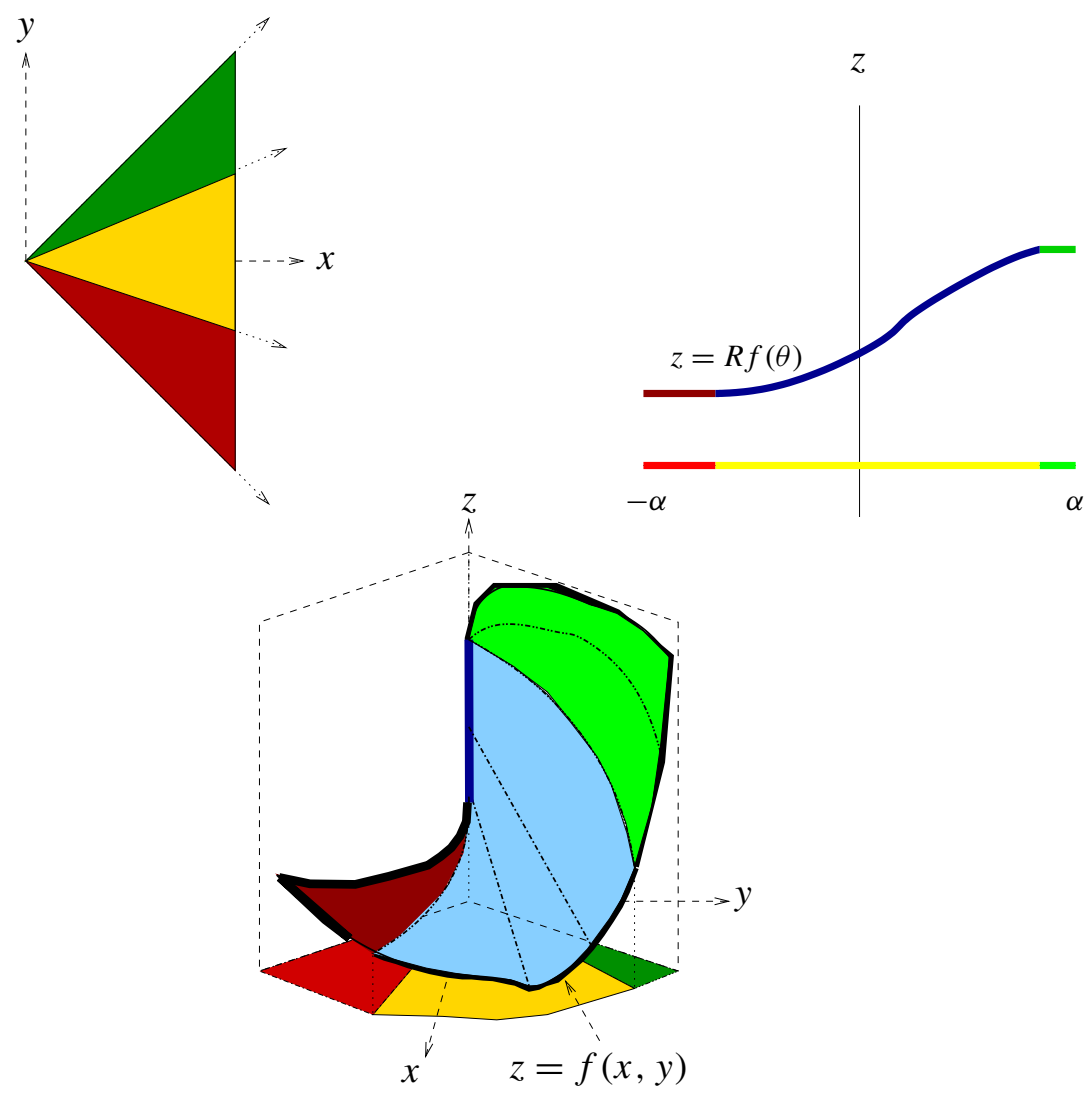

Figure 12. Radial limits: Side fans at a convex corner.

Now we wish to determine the exact sizes of the side fans (illustrated in Figure 12) when $\left(\gamma_{1}, \gamma_{2}\right) \in D_{1}^{ \pm} \cup D_{2}^{ \pm}$. From [LS a], Theorems 1 and 2, we know that if $f$ is discontinuous at $\mathcal{O}$, then $R f$ and the limits at $\mathcal{O}$ of the Gauss map behave in the following ways; here $\mathscr{I}$ denotes a countable subset of the appropriate interval(s) and $\omega(\theta)=(\cos \theta, \sin \theta, 0)$ for $\theta \in \mathbb{R}$.

Case (I)

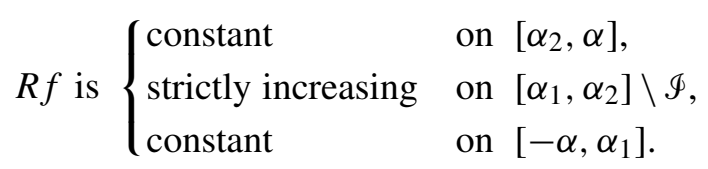

This case can only occur when $\left(\gamma_{1}, \gamma_{2}\right) \in R \cup D_{2}^{+} \cup D_{1}^{ \pm}$. Theorem 2 of [LS a] implies $\alpha_{2} \leq \alpha-\gamma_{1}$ and $\alpha_{1} \geq-\alpha+\pi-\gamma_{2}$. If $\beta \in\left(\alpha_{1}, \alpha_{2}\right)$ then $\vec{m}_{f}(\beta)=\omega\left(\beta+\frac{\pi}{2}\right)$.

Case (D)

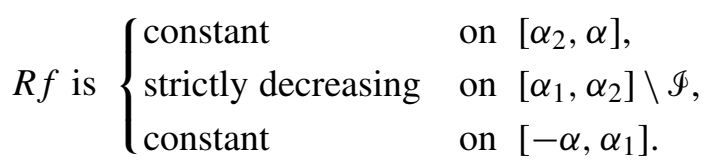


This case can only occur when $\left(\gamma_{1}, \gamma_{2}\right) \in R \cup D_{2}^{-} \cup D_{1}^{ \pm}$. Theorem 2 of [LS a] implies $\alpha_{2} \leq \alpha-\pi+\gamma_{1}$ and $\alpha_{1} \geq-\alpha+\gamma_{2}$. If $\beta \in\left(\alpha_{1}, \alpha_{2}\right)$, then $\vec{m}_{f}(\beta)=\omega\left(\beta-\frac{\pi}{2}\right)$.

Case (DI) There exists $\theta_{0} \in\left(-\alpha+\gamma_{2}, \alpha-\gamma_{1}-\pi\right)$ such that

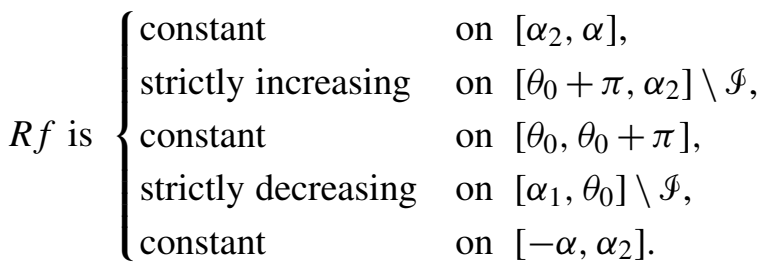

This case can only occur when $\left(\gamma_{1}, \gamma_{2}\right) \in D_{1}^{+}$. Theorem 2 of [LS a] implies $\alpha_{2} \leq$ $\alpha-\gamma_{1}$ and $\alpha_{1} \geq-\alpha+\gamma_{2}$. If $\beta \in(-\alpha, \alpha)$, then

$$
\vec{m}_{f}(\beta)= \begin{cases}\omega\left(\beta+\frac{\pi}{2}\right) & \text { if } \beta \in\left(\theta_{0}+\pi, \alpha_{2}\right), \\ \omega\left(\theta_{0}-\frac{\pi}{2}\right) & \text { if } \beta \in\left[\theta_{0}, \theta_{0}+\pi\right], \\ \omega\left(\beta-\frac{\pi}{2}\right) & \text { if } \beta \in\left(\alpha_{1}, \theta_{0}\right) .\end{cases}
$$

Case (ID) There exists $\theta_{0} \in\left(-\alpha+\pi-\gamma_{2}, \alpha+\gamma_{1}-2 \pi\right)$ such that

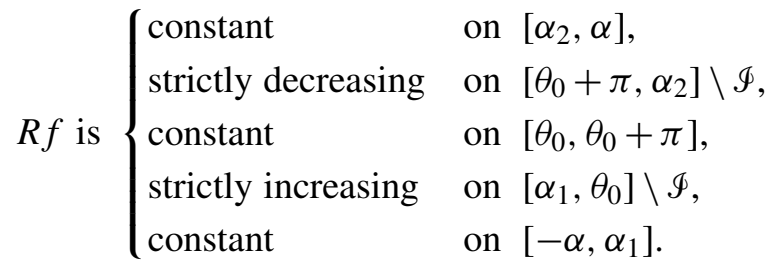

This case can only occur when $\left(\gamma_{1}, \gamma_{2}\right) \in D_{1}^{-}$. Theorem 2 of [LS a] implies $\alpha_{2} \leq$ $\alpha-\pi+\gamma_{1}$ and $\alpha_{1} \geq-\alpha+\pi-\gamma_{2}$. If $\beta \in(-\alpha, \alpha)$, then

$$
\vec{m}_{f}(\beta)= \begin{cases}\omega\left(\beta-\frac{\pi}{2}\right) & \text { if } \beta \in\left(\theta_{0}+\pi, \alpha_{2}\right), \\ \omega\left(\theta_{0}+\frac{\pi}{2}\right) & \text { if } \beta \in\left[\theta_{0}, \theta_{0}+\pi\right], \\ \omega\left(\beta+\frac{\pi}{2}\right) & \text { if } \beta \in\left(\alpha_{1}, \theta_{0}\right) .\end{cases}
$$

Theorem 3.2. Let $\Omega$ and $\gamma$ be as in Section 1, with $\alpha \in\left[\frac{\pi}{2}, \pi\right]$. For some $\rho \in(0,1)$ and $\delta>0$, suppose $f \in C^{2}(\Omega) \cap C^{1, \rho}(B(\mathcal{O}, \delta) \cap \bar{\Omega} \backslash\{\mathcal{O}\})$ is a bounded solution of (3)-(4) with $|H|_{\infty}<\infty$. Suppose (5) holds, $\gamma_{1}, \gamma_{2} \in(0, \pi)$ and $\left(\gamma_{1}, \gamma_{2}\right) \in D_{1}^{ \pm} \cup D_{2}^{ \pm}$. Then:

(i) In Case (I), $\alpha_{1}=-\alpha+\pi-\gamma_{2}$ and $\alpha_{2}=\alpha-\gamma_{1}$.

(ii) In Case (D), $\alpha_{1}=-\alpha+\gamma_{2}$ and $\alpha_{2}=\alpha-\pi+\gamma_{1}$.

(iii) In Case (DI), $\alpha_{1}=-\alpha+\gamma_{2}$ and $\alpha_{2}=\alpha-\gamma_{1}$.

(iv) In Case (ID), $\alpha_{1}=-\alpha+\pi-\gamma_{2}$ and $\alpha_{2}=\alpha-\pi+\gamma_{1}$. 
Proof. Suppose $\left(\gamma_{1}, \gamma_{2}\right) \in D_{2}^{ \pm}$; the argument is the same when $\alpha<\pi / 2$ and when $\alpha \geq \pi / 2$. Let us assume $\left(\gamma_{1}, \gamma_{2}\right) \in D_{2}^{+}$; hence Case (I) holds. Then Figure 5 illustrates the conclusions of Theorem 2.1 of [L] and Theorem 2.4. Suppose there exists $\alpha_{2}<\alpha-\gamma_{1}$ (and $\alpha_{1} \geq-\alpha+\pi-\gamma_{2}$ ) such that

$$
R f \text { is } \begin{cases}\text { constant } & \text { on }\left[\alpha_{2}, \alpha\right], \\ \text { strictly increasing } & \text { on }\left[\alpha_{1}, \alpha_{2}\right] \backslash \mathscr{I}, \\ \text { constant } & \text { on }\left[-\alpha, \alpha_{1}\right] .\end{cases}
$$

If we define

$$
\Omega^{\prime}=\left\{(r \cos \beta, r \sin \beta) \in \Omega: 0<r<\delta, \alpha_{2}<\beta<\pi\right\}
$$

for $\delta>0$ sufficiently small, then $f \in C^{0}\left(\overline{\Omega^{\prime}}\right)$ and we may apply the arguments in the proof of Theorem 2.1, using $\Omega^{\prime}$ as our domain, to obtain a contradiction. If $\alpha_{1}>-\alpha+\pi-\gamma_{2}$, a similar argument yields a contradiction.

Now suppose $\left(\gamma_{1}, \gamma_{2}\right) \in D_{1}^{+}$, Case (I) holds and there exist $\alpha_{2}<\alpha-\gamma_{1}$ and $\alpha_{1} \geq-\alpha+\pi-\gamma_{2}$ such that

$$
R f \text { is } \begin{cases}\text { constant } & \text { on }\left[\alpha_{2}, \alpha\right], \\ \text { strictly increasing } & \text { on }\left[\alpha_{1}, \alpha_{2}\right] \backslash \mathscr{I}, \\ \text { constant } & \text { on }\left[-\alpha, \alpha_{1}\right] .\end{cases}
$$

Let $\theta_{1} \in\left(-\alpha,-\alpha_{1}+\pi-\gamma_{2}\right)$ and $\theta_{2} \in\left(\alpha-\gamma_{1}, \alpha\right)$. By choosing $\delta_{0}>0$ small, we may assume $\Omega^{*}=\left\{(r \cos \theta, r \sin \theta): 0<r<\delta_{0}, \theta_{1}<\theta<\theta_{2}\right\}$ is a subset of $\Omega$. Set $\Omega^{\prime}=\left\{(r \cos \beta, r \sin \beta): 0<r<\delta_{0}, \alpha_{2}<\theta<\theta_{2}\right\}$ and notice that $f \in C^{0}\left(\overline{\Omega^{\prime}}\right)$. Now Theorem 2.5, Lemma 3.1 and the fact that

$$
\lim _{j \rightarrow \infty} \vec{n}_{f}\left(x_{j}\right)=\omega\left(\beta+\frac{\pi}{2}\right)
$$

when $\beta \in\left(\alpha_{1}, \alpha_{2}\right)$ and $\left(x_{j}\right) \in s(\Omega)$ such that $\lim _{j \rightarrow \infty} x_{j} /\left|x_{j}\right|=(\cos \beta, \sin \beta)$ implies that

$$
\Pi_{f}\left(\Omega^{*}\right) \subset\left\{\omega(\theta): \beta \in\left[\theta_{1}, \theta_{2}\right],(\beta, \theta) \in \mathscr{F}_{C}\right\}
$$

and $\vec{m}_{f}(\cdot) \in C^{0}((-\alpha, \alpha))$. If $\vec{m}_{f}\left(\alpha_{2}\right) \neq \omega\left(\alpha-\gamma_{1}+\frac{\pi}{2}\right)$, then we may apply the arguments in the proof of Theorem 2.1, using $\Omega^{\prime}$ as our domain, to get a contradiction. If $\vec{m}_{f}\left(\alpha_{2}\right)=\omega\left(\alpha-\gamma_{1}+\frac{\pi}{2}\right)$, then $\vec{m}_{f}$ is discontinuous at $\alpha_{2}$, which is a contradiction. Therefore $\alpha_{2}=\alpha-\gamma_{1}$. The argument that $\alpha_{1}=-\alpha+\pi-\gamma_{2}$ is similar.

The proof of the theorem when $\left(\gamma_{1}, \gamma_{2}\right) \in D_{1}^{+}$and one of Cases (D), (DI) or (ID) occurs follows in a similar manner. The situation where $\left(\gamma_{1}, \gamma_{2}\right) \in D_{1}^{-}$follows from this. 


\section{Continuity of the Gauss map}

Notice that Lemma 3.1 and the proof of Theorem 3.2 imply that the (nonparametric) Gauss map is continuous on $\mathscr{Y}_{f} \cap\left(\overline{\Omega_{\epsilon}} \times \mathbb{R}\right)$ for each $\epsilon>0$ and, in each case, we have:

(I): $\lim _{\beta \rightarrow \alpha} \vec{m}_{f}(\beta)=\omega\left(\alpha_{2}+\pi / 2\right)$ and $\lim _{\beta \rightarrow-\alpha} \vec{m}_{f}(\beta)=\omega\left(\alpha_{1}+\pi / 2\right)$.

(D): $\lim _{\beta \rightarrow \alpha} \vec{m}_{f}(\beta)=\omega\left(\alpha_{2}-\pi / 2\right)$ and $\lim _{\beta \rightarrow-\alpha} \vec{m}_{f}(\beta)=\omega\left(\alpha_{1}-\pi / 2\right)$.

(DI): $\lim _{\beta \rightarrow \alpha} \vec{m}_{f}(\beta)=\omega\left(\alpha_{2}+\pi / 2\right)$ and $\lim _{\beta \rightarrow-\alpha} \vec{m}_{f}(\beta)=\omega\left(\alpha_{1}-\pi / 2\right)$.

(ID): $\lim _{\beta \rightarrow \alpha} \vec{m}_{f}(\beta)=\omega\left(\alpha_{2}-\pi / 2\right)$ and $\lim _{\beta \rightarrow-\alpha} \vec{m}_{f}(\beta)=\omega\left(\alpha_{1}+\pi / 2\right)$.

In order to conclude that the Gauss map is in $C^{0}\left(\mathscr{Y}_{f} \cap(B(\mathbb{O}, \delta) \times \mathbb{R}): S_{-}^{2}\right)$, it would be sufficient to blow up the graph of $u(x)=f(x)-R f(\alpha)$ (or $u(x)=f(x)-R f(-\alpha)$ ) about $(0,0,0)$ tangent to $\partial^{+} \Omega$ (or $\partial^{-} \Omega$ respectively) and know that a subsequence converges to an appropriate cone. If one is willing to accept this hypothesis, then the claim that the Gauss map is in $C^{0}\left(\mathscr{Y}_{f} \cap(B(\mathcal{O}, \delta) \times \mathbb{R}): S_{-}^{2}\right)$ can be proven.

Hypothesis $(\mathbf{B} \pm)$. For all $\left(x_{j}\right) \in s(\Omega)$ with $\lim _{j \rightarrow \infty} x_{j} /\left|x_{j}\right|=(\cos ( \pm \alpha), \sin ( \pm \alpha))$, there is a subsequence $\left(x_{j_{k}}\right)$ and a function $u_{\infty}: \Omega_{\infty} \rightarrow[-\infty, \infty]$ such that the subgraph $U_{\infty}=\left\{(x, t) \in \Omega_{\infty} \times \mathbb{R}: t<u_{\infty}(x)\right\}$ of $u_{\infty}$ is a cone with respect to $(0,0,0)$, there exists $\vec{\xi} \in S_{-}^{2}$ such that $\lim _{k \rightarrow \infty} \vec{n}\left(x_{j_{k}}\right)=\vec{\xi}=\left(\xi_{1}, \xi_{2}, \xi_{3}\right)$,

$$
\lim _{k \rightarrow \infty} \operatorname{dist}\left(\left\{\left(x, u_{j_{k}}(x)\right) \in \Omega_{j_{k}}(\delta, b)\right\}, \partial U_{\infty} \cap \Omega_{j_{k}}(\delta, b)\right)=0
$$

for each $\delta>0$ and $b>0$, where $\epsilon_{j}=\left|x_{j}\right|, u_{j}(x)=\left(f\left(\epsilon_{j} x\right)-R f( \pm \alpha)\right) / \epsilon_{j}$ and $\Omega_{j}(\delta, b)=\left\{(x, t) \in \mathbb{R}^{3}: x \in B(\mathbb{O}, \delta), \epsilon_{j} x \in \Omega, t \in(-b, b)\right\}$ for $j \in \mathbb{N}$, and

(a) if $\xi_{3}<0$, then $\partial U_{\infty}=\pi_{1} \cap\left(\overline{\Omega_{\infty}} \times \mathbb{R}\right), \pi_{1}$ is a nonvertical plane with downward unit normal $\vec{\xi} \in S_{-}^{2}, \vec{\xi}$ makes an angle of $\gamma_{1}$ with the exterior unit normal to $\partial^{+} \Omega_{\infty} \times \mathbb{R}$ and an angle of $\gamma_{2}$ with the exterior unit normal to $\partial^{-} \Omega_{\infty} \times \mathbb{R}$ and $\vec{n}_{u_{j_{k}}} \rightarrow \vec{\xi}$ uniformly on compacta in $\Omega \times \mathbb{R}$ as $k \rightarrow \infty$,

(b) if $\xi_{3}=0$, then $\partial U_{\infty}=\partial \mathscr{P} \cap\left(\overline{\Omega_{\infty}} \times \mathbb{R}\right), \mathscr{P}=\{(r \cos \theta, r \sin \theta): r>0, \theta \in \Theta\}$ and, for each $x \in \partial \mathscr{P} \cap \Omega_{\infty}, \vec{n}_{u_{j_{k}}}(x) \rightarrow \vec{\xi}(x) \times\{0\}$, where $\mathscr{P}=\left\{x \in \Omega_{\infty}: u_{\infty}(x)=\infty\right\}$, $\vec{\xi}(x)$ is the interior (with respect to $\mathscr{P}$ ) unit normal vector to $\partial \mathscr{P}$ at $x$ and $\Theta$ is one of the following sets: $\left(\alpha-\gamma_{1}, \alpha\right),\left(-\alpha,-\alpha+\gamma_{2}\right),\left(-\alpha+\pi-\gamma_{2}, \alpha-\pi+\gamma_{1}\right)$ (provided $\left.\left(\alpha-\pi+\gamma_{1}\right)-\left(-\alpha+\pi-\gamma_{2}\right) \geq \pi\right)$ or $\left(-\alpha,-\alpha+\gamma_{2}\right) \cup\left(\alpha-\gamma_{1}, \alpha\right)$ (provided $\left.\left(\alpha-\gamma_{1}\right)-\left(-\alpha+\gamma_{2}\right) \geq \pi\right)$.

Theorem 4.1. Let $\Omega$ and $\gamma$ be as in Section 1. For some $\rho \in(0,1)$ and $\delta>0$, suppose $f \in C^{2}(\Omega) \cap C^{1, \rho}(B(\mathcal{O}, \delta) \cap \bar{\Omega} \backslash\{\mathcal{O}\})$ is a bounded solution of (3)-(4) with $|H|_{\infty}<\infty$. Suppose (5) holds, $\gamma_{1}, \gamma_{2} \in(0, \pi)$ and either $\alpha<\pi / 2$ and $\left(\gamma_{1}, \gamma_{2}\right)$ is in $D_{2}^{ \pm}$or $\alpha \geq \pi / 2$ and $\left(\gamma_{1}, \gamma_{2}\right)$ is in $D_{1}^{ \pm} \cup D_{2}^{ \pm}$. Suppose that Hypotheses $(\mathrm{B} \pm)$ are true. Then $\vec{n}_{f}: \mathscr{Y}_{f} \times(\Omega \times \mathbb{R})$ extends to be continuous on $\left.\mathscr{Y}_{f} \cap(B(O, \delta) \times \mathbb{R})\right)$ and $N \in C^{0}(E \cup\{(u, v) \in \partial E: G(u, v) \in \partial \Omega \cap B(\mathcal{O}, \delta)\})$. 
The proof of this theorem will follow from the information at the beginning of this section about the behavior of $\vec{m}_{f}$ and from Lemmas 4.2-4.5. Set

$$
\begin{gathered}
C(x)=\left\{X \in S_{-}^{2}: X \cdot v(x)=\cos \gamma(x)\right\}, \\
\Gamma_{1}=\left\{X \in S_{-}^{2}: X \cdot\left(\cos \left(\alpha+\frac{\pi}{2}\right), \sin \left(\alpha+\frac{\pi}{2}\right), 0\right)=\cos \gamma_{1}\right\}, \\
\Gamma_{2}=\left\{X \in S_{-}^{2}: X \cdot\left(\cos \left(-\alpha-\frac{\pi}{2}\right), \sin \left(-\alpha-\frac{\pi}{2}\right), 0\right)=\cos \gamma_{2}\right\}, \\
\vec{\xi}_{A}=\omega\left(\alpha-\gamma_{1}+\frac{\pi}{2}\right) \in \Gamma_{1}, \quad \vec{\xi}_{B}=\omega\left(\alpha+\gamma_{1}-\frac{3 \pi}{2}\right) \in \Gamma_{1}, \\
\vec{\xi}_{C}=\omega\left(-\alpha-\gamma_{2}+\frac{3 \pi}{2}\right) \in \Gamma_{2}, \quad \vec{\xi}_{D}=\omega\left(-\alpha+\gamma_{2}-\frac{\pi}{2}\right) \in \Gamma_{2}, \\
\Omega_{\infty}=\{(r \cos (\theta), r \sin (\theta)): r>0,-\alpha<\theta<\alpha\}, \\
\Sigma_{\infty}^{1}=\{(r \cos (\alpha), r \sin (\alpha)): r>0\}, \quad \Sigma_{\infty}^{2}=\{(r \cos (\alpha),-r \sin (\alpha)): r>0\}, \\
v_{\infty}^{+}=\left(\cos \left(\alpha+\frac{\pi}{2}\right), \sin \left(\alpha+\frac{\pi}{2}\right), 0\right) \quad \text { and } \quad v_{\infty}^{-}=\left(\cos \left(-\alpha-\frac{\pi}{2}\right), \sin \left(-\alpha-\frac{\pi}{2}\right), 0\right) .
\end{gathered}
$$

Lemma 4.2. Let $\Omega$ and $\gamma$ be as in Section 1. For some $\rho \in(0,1)$ and $\delta>0$, suppose $f \in C^{2}(\Omega) \cap C^{1, \rho}(B(\mathbb{O}, \delta) \cap \bar{\Omega} \backslash\{\mathcal{O}\})$ is a bounded solution of (3)-(4) with $|H|_{\infty}<\infty$. Suppose (5) holds, $\gamma_{1}, \gamma_{2} \in(0, \pi)$ and either $\alpha<\pi / 2$ and $\left(\gamma_{1}, \gamma_{2}\right)$ is in $D_{2}^{+}$, so that $R f$ behaves as in Case (I), or $\alpha \geq \pi / 2$ and $\left(\gamma_{1}, \gamma_{2}\right)$ is in $D_{1}^{ \pm} \cup D_{2}^{+}$ and $R f$ behaves as in Case (I) or Case (DI). Assume Hypothesis (B+) is true. Then

$$
\lim _{j \rightarrow \infty} \vec{n}\left(x_{j}\right)=\left(\cos \left(\alpha-\gamma_{1}+\pi / 2\right), \sin \left(\alpha-\gamma_{1}+\pi / 2\right), 0\right)=\vec{\xi}_{A}
$$

for every $\left(x_{j}\right) \in s(\Omega)$ with $\lim _{j \rightarrow \infty} x_{j} /\left|x_{j}\right|=(\cos \alpha, \sin \alpha)$.

Proof. Since $\gamma(x) \rightarrow \gamma_{1}$ and $v(x) \rightarrow v_{\infty}^{+}$as $x \in \partial^{+} \Omega$ converges to $\mathbb{O}$, we see that $\operatorname{dist}\left(C(x), \Gamma_{1}\right) \rightarrow 0$ as $x \in \partial^{+} \Omega$ converges to $\mathrm{O}$. Similarly, $\gamma(x) \rightarrow \gamma_{2}, v(x) \rightarrow v_{\infty}^{-}$ and $\operatorname{dist}\left(C(x), \Gamma_{2}\right) \rightarrow 0$ as $x \in \partial^{-} \Omega$ converges to $\mathbb{O}$. Thus

$$
\operatorname{dist}\left(\vec{n}\left(x^{+}(s)\right), \Gamma_{1}\right) \rightarrow 0 \quad \text { and } \quad \operatorname{dist}\left(\vec{n}\left(x^{-}(s)\right), \Gamma_{2}\right) \rightarrow 0
$$

as $s \rightarrow 0+$.

Suppose that $\left(x_{j}\right) \in s(\Omega)$ with $\lim _{j \rightarrow \infty} x_{j} /\left|x_{j}\right|=(\cos \alpha, \sin \alpha)$; then there is a subsequence, still denoted $\left(x_{j}\right)$, and $\vec{\xi} \in S_{-}^{2}$ such that $\lim _{j \rightarrow \infty} \vec{n}\left(x_{j}, y_{j}\right) \rightarrow \vec{\xi}$. Notice that $\vec{\xi} \in \Gamma_{1}$ since $f \in C^{1, \rho}(B(\mathbb{O}, \delta) \cap \bar{\Omega} \backslash\{\mathcal{O}\})$ and (17) holds.

Assume first that $\vec{\xi}=\left(\xi_{1}, \xi_{2}, \xi_{3}\right)$ with $\xi_{3}<0$. For each $j \in \mathbb{N}$, define $\epsilon_{j}=\left|x_{j}\right|$, $\Omega_{j}=\left\{x \in \mathbb{R}^{2}: \epsilon_{j} x \in \Omega\right\}$ and $u_{j} \in C^{\infty}\left(\Omega_{j}\right) \cap C^{1}\left(\overline{\Omega_{j}} \backslash\{\mathbb{O}\}\right)$ by

$$
u_{j}(x)=\frac{1}{\epsilon_{j}}\left(f\left(\epsilon_{j} x\right)-R f(\alpha)\right) .
$$


Let $\gamma_{j}$ be defined on $\partial \Omega_{j} \backslash\{\mathcal{O}\}$ by $\gamma_{j}(x)=\gamma\left(\epsilon_{j} x\right)$ and let $v_{j}$ denote the outward unit normal to $\partial \Omega_{j}$. Then $u_{j}$ satisfies the prescribed mean curvature problem

$$
\begin{aligned}
& N u_{j}(x)=\epsilon_{j} H\left(\epsilon_{j} x, f\left(\epsilon_{j} x\right)\right), \quad x \in \Omega_{j}, \\
& T f_{j} \cdot v_{j}=\cos \gamma_{j} \quad \text { on } \partial \Omega_{j} \backslash\{\mathcal{O}\} .
\end{aligned}
$$

Hypothesis $(\mathrm{B}+)$ implies that there is a nonvertical plane $\pi_{1}$ with downward unit normal $\vec{\xi}$ which meets $\partial^{+} \Omega_{\infty}$ in an angle of $\gamma_{1}$ and $\partial^{-} \Omega_{\infty}$ in an angle of $\gamma_{2}$ in the sense described in (a); however this is impossible since $\left(\gamma_{1}, \gamma_{2}\right) \in D_{1}^{ \pm} \cup D_{2}^{ \pm}$. Thus $\xi_{3}=0$ and so either $\vec{\xi}=\vec{\xi}_{A}$ or $\vec{\xi}=\vec{\xi}_{B}$. The intermediate value theorem implies that

$$
\begin{array}{ll}
\text { if } \vec{\xi}=\vec{\xi}_{A}, & \text { then } \vec{n}(x) \rightarrow \vec{\xi}_{A} \text { as } x \in \partial^{+} \Omega \text { converges to } \mathbb{O}, \\
\text { if } \vec{\xi}=\vec{\xi}_{B}, & \text { then } \vec{n}(x) \rightarrow \vec{\xi}_{B} \text { as } x \in \partial^{+} \Omega \text { converges to } \mathbb{O} .
\end{array}
$$

Suppose (22) holds. Notice then that

$$
\lim _{s \rightarrow 0+} \frac{d}{d s} f^{+}(s)=-\infty
$$

since $(\cos \alpha, \sin \alpha, 0) \cdot \vec{\xi}_{B}=-\sin \left(\gamma_{1}\right)<0$, and so $f^{+}(s)=f\left(x^{+}(s)\right)$ is a strictly decreasing function of $s$ for $0 \leq s \leq s_{0}$, where $s_{0}>0$ is sufficiently small. Since $\vec{m}_{f}(\beta)=\omega\left(\alpha-\gamma_{1}+\frac{\pi}{2}\right)$ when $\beta \in\left[\alpha-\gamma_{1}, \alpha\right)$, we have

$$
\lim _{r \rightarrow 0+} \nabla f(r \cos \beta, r \sin \beta) \cdot(\cos \beta, \sin \beta)=+\infty \quad \text { for } \beta \in\left(\alpha-\gamma_{1}, \alpha\right) .
$$

Since $R f$ behaves as in Case (I) or (DI), we have

$$
R f(\beta)<R f(\alpha)=f^{+}(0) \quad \text { if } \beta \in\left[\alpha-\pi, \alpha-\gamma_{1}\right) .
$$

Let $\Omega_{H}$ be the connected component of

$$
\{(r \cos \beta, r \sin \beta) \in \Omega: r>0, \alpha-\pi<\beta<5 \pi / 4\}
$$

that contains $\left\{(r \cos \beta, r \sin \beta): 0<r<\delta, \alpha-\gamma_{1}<\beta<\alpha-\epsilon\right\}$ for sufficiently small $\epsilon, \delta>0$. Consider the $k=f^{+}(0)(=R f(\alpha))$ level set of $f$ in $\Omega_{H}$. From (23), we see that there is a component $C$ of $\left\{x \in \Omega_{H}: f(x)<k\right\}$ whose boundary contains $\partial^{+} \Omega \cap B(\mathcal{O}, \tau)$ for $\tau>0$ sufficiently small; let $c_{\alpha}$ be the component of $\Omega_{H} \cap \partial C$ whose closure contains 0 . Then (23) and (24) imply that for every $\beta_{1}<\alpha$ and $\beta_{2}>\alpha$, there exists $\epsilon>0$ such that

$$
c_{\alpha} \cap B(\mathcal{O}, \epsilon) \subset\left\{(r \cos \theta, r \sin \theta): 0<r<\epsilon, \beta_{1}<\theta<\beta_{2}\right\} ;
$$

in this sense, $c_{\alpha}$ is tangent to $\theta=\alpha$ at $\mathbb{O}$. Similarly, using (24) and (25), we see that there is a $k$-level curve of $f$, denoted $c_{\alpha-\gamma_{1}}$, which is tangent to $\theta=\alpha-\gamma_{1}$ at 0 in the sense that for every $\beta_{1}<\alpha-\gamma_{1}$ and $\beta_{2}>\alpha-\gamma_{1}$, there exists $\epsilon>0$ such that

$$
c_{\alpha-\gamma_{1}} \cap B(\mathcal{O}, \epsilon) \subset\left\{(r \cos \theta, r \sin \theta): 0<r<\epsilon, \beta_{1}<\theta<\beta_{2}\right\} .
$$


Now pick $\tau>0$ small enough that the region bounded by $c_{\alpha}, c_{\alpha-\gamma_{1}}$ and $\{r=\tau\}$ is well-defined, connected and simply connected; let us rotate this region about 0 through an angle $\left(\pi+\gamma_{1}\right) / 2-\alpha$ and denote this open set as $\Omega^{\tau}$, so that $\partial \Omega^{\tau}$ is tangent to $\theta=\left(\pi \pm \gamma_{1}\right) / 2$ at $\mathcal{O}$. Notice that $\tilde{f}=f \circ R^{-1} \in C^{0}\left(\overline{\Omega^{\tau}}\right)$ if $R$ denotes the rotation above. We will let a particular portion of a suitable nodoid be the graph of a comparison function $h$ over a domain $U^{\tau} \subset \Omega^{\tau}$ with $B(\mathcal{O}, \epsilon) \cap U^{\tau}=B(\mathcal{O}, \epsilon) \cap \Omega^{\tau}$ for some $\epsilon>0$. Now $\partial U^{\tau}$ will be consist of two disjoint, connected curves, $\partial_{1} U^{\tau} \subset \partial \Omega^{\tau} \backslash\{r=\tau\}$ and $\partial_{2} U^{\tau}$, with $\mathcal{O} \in \partial_{1} U^{\tau}$ and $\mathcal{O} \notin \overline{\partial_{2} U^{\tau}}$. The comparison function $h \in C^{0}\left(\overline{U^{\tau}}\right) \cap C^{1}\left(U^{\tau} \cup \partial_{1} U^{\tau}\right)$ will have the properties $h(\mathcal{O})=k, \frac{\partial h}{\partial x_{2}}(\mathbb{O})<\infty$, $h \geq k=\tilde{f}$ on $\partial_{1} U^{\tau}, N h \leq \inf _{x \in \Omega} N f(x)$ on $U^{\tau}$ and $\frac{\partial h}{\partial \eta}=\nabla f \cdot \eta=+\infty$ on $\partial_{2} U^{\tau}$, where $\eta$ is the exterior unit normal to $\partial_{2} U^{\tau}$. The comparison principle then implies $\tilde{f} \leq h$ on $\overline{U^{\tau}}$. This yields a contradiction of (22) since (24) implies

$$
\lim _{x_{2} \downarrow 0} \frac{\partial \tilde{f}}{\partial x_{2}}\left(0, x_{2}\right)=+\infty
$$

and the facts that $\tilde{f}(\mathbb{O})=h(\mathbb{O}), \frac{\partial h}{\partial x_{2}}(\mathbb{O})<\infty$ and $\tilde{f} \leq h$ imply

$$
\liminf _{x_{2} \downarrow 0} \frac{\partial \tilde{f}}{\partial x_{2}}\left(0, x_{2}\right)<\infty .
$$

This implies (21) holds and completes the proof of Lemma 4.2 except for the construction of the comparison function $h$.

Let $\mathscr{C}$ be a nodary in $\left\{x \in \mathbb{R}^{2}: x_{2}>0\right\}$ which, when rotated about the $x_{1}$-axis, generates a nodoid in $\mathbb{R}^{3}$ with constant mean curvature $H_{D}=|H|_{\infty}$, which we assume is positive; if not, set $H_{D}=1$. (See, for example, [Eells 1987; Mladenov 2002; Rossman 2005] for discussions of Delaunay surfaces and nodoids.) Let the minimal and maximal radii of the nodary be $r_{0}$ and $R_{0}$ respectively, so that $r_{0} \leq x_{2} \leq R_{0}$ whenever $\left(x_{1}, x_{2}\right) \in \mathscr{C}$; we will assume $\left(0, r_{0}\right) \in \mathscr{C}$. Now let $\mathscr{D} \subset \mathscr{C}$ be the particular open inner loop of the nodary which contains $\left(0, r_{0}\right)$ (i.e., $\left(0, r_{0}\right) \in \mathscr{D}$ and $\mathscr{D}$ does not contain endpoints); notice that the unit normal to the nodary at the endpoints of $\overline{\mathscr{D}}$ are parallel to the axis of rotation of the nodoid and the surface

$$
S_{\mathscr{D}}=\left\{\left(x_{1}, x_{2} \cos \theta, x_{2} \sin \theta\right):\left(x_{1}, x_{2}\right) \in \mathscr{D},-\pi \leq \theta \leq 0\right\}
$$

obtained by partially rotating $\mathscr{D}$ about the $x_{1}$-axis has constant mean curvature $-H_{D}$ with respect to its upward unit normal.

Now fix $t, 0<t<r_{0}$, large enough that $\Phi_{1}=\left\{\left(x_{1}, x_{2}+t\right): x \in \partial \Omega^{\tau} \cap R\left(c_{\alpha}\right)\right\}$ and $\Phi_{2}=\left\{\left(x_{1}, x_{2}+t\right): x \in \partial \Omega^{\tau} \cap R\left(c_{\alpha-\gamma_{1}}\right)\right\}$ both intersect $\mathscr{D}$. Let $\Sigma$ denote the component of $\bar{\Phi}_{1} \cup \bar{\Phi}_{2} \backslash \mathscr{D}$ that contains $(0, t)$ and let $W$ be the region bounded by $\Sigma$ and $\mathscr{D}$. Set $W^{\tau}=\left\{\left(x_{1}, x_{2}\right):\left(x_{1}, x_{2}+t\right) \in W\right\}, \partial_{1} W^{\tau}=\left\{\left(x_{1}, x_{2}\right):\left(x_{1}, x_{2}+t\right) \in \Sigma\right\}$ and $\partial_{2} W^{\tau}=\partial W^{\tau} \backslash \partial_{1} W^{\tau}$. Notice that $\partial_{2} W^{\tau} \subset\left\{\left(x_{1}, x_{2}\right):\left(x_{1}, x_{2}+t\right) \in \mathscr{D}\right\}$. Now 
define $h \in C^{0}\left(\overline{W^{\tau}}\right) \cap C^{1}\left(W^{\tau} \cup \partial_{1} W^{\tau}\right)$ by

$$
h\left(x_{1}, x_{2}\right)=w\left(x_{1}, x_{2}+t\right)-w(0, t)+k
$$

for $x \in \overline{W^{\tau}}$, where $w: \mathscr{D} \rightarrow \mathbb{R}$ such that $S_{\mathscr{D}}$ is the graph of $w$. It follows that $h$ has the properties mentioned previously.

In a similar manner, we can prove each of the following lemmas.

Lemma 4.3. Let $\Omega$ and $\gamma$ be as in Section 1. For some $\rho \in(0,1)$ and $\delta>0$, suppose $f \in C^{2}(\Omega) \cap C^{1, \rho}(B(\mathbb{O}, \delta) \cap \bar{\Omega} \backslash\{\mathcal{O}\})$ is a bounded solution of (3)-(4) with $|H|_{\infty}<\infty$. Suppose (5) holds, $\gamma_{1}, \gamma_{2} \in(0, \pi)$ and either $\alpha<\pi / 2$ and $\left(\gamma_{1}, \gamma_{2}\right)$ is in $D_{2}^{-}$, so that $R f$ behaves as in Case (D), or $\alpha \geq \pi / 2$ and $\left(\gamma_{1}, \gamma_{2}\right)$ is in $D_{1}^{ \pm} \cup D_{2}^{-}$ and $R f$ behaves as in Case (D) or Case (ID). Assume Hypothesis (B+) is true. Then

$$
\lim _{j \rightarrow \infty} \vec{n}\left(x_{j}\right)=\left(\cos \left(\alpha+\gamma_{1}-3 \pi / 2\right), \sin \left(\alpha+\gamma_{1}-3 \pi / 2\right), 0\right)
$$

for every $\left(x_{j}\right) \in s(\Omega)$ with $\lim _{j \rightarrow \infty} x_{j} /\left|x_{j}\right|=(\cos \alpha, \sin \alpha)$.

Lemma 4.4. Let $\Omega$ and $\gamma$ be as in Section 1. For some $\rho \in(0,1)$ and $\delta>0$, suppose $f \in C^{2}(\Omega) \cap C^{1, \rho}(B(\mathcal{O}, \delta) \cap \bar{\Omega} \backslash\{\mathcal{O}\})$ is a bounded solution of (3)-(4) with $|H|_{\infty}<\infty$. Suppose (5) holds, $\gamma_{1}, \gamma_{2} \in(0, \pi)$ and either $\alpha<\pi / 2$ and $\left(\gamma_{1}, \gamma_{2}\right)$ is in $D_{2}^{+}$, so that $R f$ behaves as in Case (I), or $\alpha \geq \pi / 2$ and $\left(\gamma_{1}, \gamma_{2}\right)$ is in $D_{1}^{ \pm} \cup D_{2}^{+}$ and $R f$ behaves as in Case (I) or Case (ID). Assume Hypothesis (B-) is true. Then

$$
\lim _{j \rightarrow \infty} \vec{n}\left(x_{j}\right)=\left(\cos \left(-\alpha-\gamma_{1}+3 \pi / 2\right), \sin \left(-\alpha-\gamma_{1}+3 \pi / 2\right), 0\right)
$$

for every $\left(x_{j}\right) \in s(\Omega)$ with $\lim _{j \rightarrow \infty} x_{j} /\left|x_{j}\right|=(\cos (-\alpha), \sin (-\alpha))$.

Lemma 4.5. Let $\Omega$ and $\gamma$ be as in Section 1. For some $\rho \in(0,1)$ and $\delta>0$, suppose $f \in C^{2}(\Omega) \cap C^{1, \rho}(B(\mathcal{O}, \delta) \cap \bar{\Omega} \backslash\{\mathcal{O}\})$ is a bounded solution of (3)-(4) with $|H|_{\infty}<\infty$. Suppose (5) holds, $\gamma_{1}, \gamma_{2} \in(0, \pi)$ and either $\alpha<\pi / 2$ and $\left(\gamma_{1}, \gamma_{2}\right)$ is in $D_{2}^{-}$, so that $R f$ behaves as in Case (D), or $\alpha \geq \pi / 2$ and $\left(\gamma_{1}, \gamma_{2}\right)$ is in $D_{1}^{ \pm} \cup D_{2}^{-}$ and $R f$ behaves as in Case (D) or Case (DI). Assume Hypothesis (B-) is true. Then

$$
\lim _{j \rightarrow \infty} \vec{n}\left(x_{j}\right)=\left(\cos \left(-\alpha+\gamma_{1}-\pi / 2\right), \sin \left(-\alpha+\gamma_{1}-\pi / 2\right), 0\right)
$$

for every $\left(x_{j}\right) \in s(\Omega)$ with $\lim _{j \rightarrow \infty} x_{j} /\left|x_{j}\right|=(\cos (-\alpha), \sin (-\alpha))$.

\section{References}

[Concus and Finn 1996] P. Concus and R. Finn, "Capillary wedges revisited", SIAM J. Math. Anal. 27:1 (1996), 56-69. MR 96m:76006 Zbl 0843.76012

[Eells 1987] J. Eells, “The surfaces of Delaunay”, Math. Intelligencer 9:1 (1987), 53-57. MR 88h: 53011 Zbl 0605.53002

[Finn 1986] R. Finn, Equilibrium capillary surfaces, Grundlehren der Mathematischen Wissenschaften 284, Springer, New York, 1986. MR 88f:49001 Zbl 0583.35002 
[Finn 1988a] R. Finn, "Comparison principles in capillarity", pp. 156-197 in Partial differential equations and calculus of variations, edited by S. Hildebrandt and R. Leis, Lecture Notes in Math. 1357, Springer, Berlin, 1988. MR 90d:53010 Zbl 0692.35006

[Finn 1988b] R. Finn, "Moon surfaces, and boundary behaviour of capillary surfaces for perfect wetting and nonwetting”, Proc. London Math. Soc. (3) 57:3 (1988), 542-576. MR 89m:49076 Zbl 0668.76019

[Finn 1996] R. Finn, "Local and global existence criteria for capillary surfaces in wedges", Calc. Var. Partial Differential Equations 4:4 (1996), 305-322. MR 97f:53005 Zbl 0872.76017

[Finn 1999] R. Finn, “Capillary surface interfaces”, Notices Amer. Math. Soc. 46:7 (1999), 770-781. MR 2000g:76033 Zbl 1194.76049

[Finn 2002a] R. Finn, "Eight remarkable properties of capillary surfaces", Math. Intelligencer 24:3 (2002), 21-33. MR 2003f:76041

[Finn 2002b] R. Finn, "Some properties of capillary surfaces", Milan J. Math. 70 (2002), 1-23. MR 2003m:53012 Zbl 1053.76009

[Jeffres and Lancaster 2007] T. Jeffres and K. Lancaster, "Vertical blow ups of capillary surfaces in $\mathbb{R}^{3}$. I. Convex corners”, Electron. J. Differential Equations 152 (2007), 24 pp. MR 2009b:49103 Zbl 1139.49041

[Jeffres and Lancaster 2008] T. Jeffres and K. Lancaster, "Vertical blow ups of capillary surfaces in $\mathbb{R}^{3}$. II. Nonconvex corners", Electron. J. Differential Equations 160 (2008), 25 pp. MR 2009j:49098 Zbl 1163.49044

[Lancaster 2010] $=$ [L] K. E. Lancaster, "A proof of the Concus-Finn conjecture", Pacific J. Math. 247:1 (2010), 75-108. MR 2011m:53011 Zbl 05809032

[Lancaster and Siegel 1996a] $=$ [LS a] K. E. Lancaster and D. Siegel, "Existence and behavior of the radial limits of a bounded capillary surface at a corner", Pacific J. Math. 176:1 (1996), 165-194. MR 98g:58030a Zbl 0866.76018

[Lancaster and Siegel 1996b] $=$ [LS b] K. E. Lancaster and D. Siegel, "Behavior of a bounded non-parametric $H$-surface near a reentrant corner”, Z. Anal. Anwendungen 15:4 (1996), 819-850. MR 97m:53011 Zbl 0866.35046

[Mladenov 2002] I. M. Mladenov, "Delaunay surfaces revisited", C. R. Acad. Bulgare Sci. 55:5 (2002), 19-24. MR 2003g:53005 Zbl 1017.53010

[Rossman 2005] W. Rossman, "The first bifurcation point for Delaunay nodoids", Experiment. Math. 14:3 (2005), 331-342. MR 2006j:53011 Zbl 1092.53010

[Shi 2006] D. Shi, "Capillary surfaces at a reentrant corner", Pacific J. Math. 224:2 (2006), 321-353. MR 2008a:58013 Zbl 1125.76013

[Tam 1986] L.-F. Tam, "Regularity of capillary surfaces over domains with corners: borderline case", Pacific J. Math. 124:2 (1986), 469-482. MR 87k:49049 Zbl 0604.49029

Received July 12, 2010. Revised July 8, 2012.

KIRK E. LANCASTER

DEPARTMENT OF MATHEMATICS AND STATISTICS

WICHITA STATE UNIVERSITY

WICHITA, KS 67260-0033

UNITED STATES

lancaster@math.wichita.edu 


\title{
PACIFIC JOURNAL OF MATHEMATICS
}

\author{
http://pacificmath.org \\ Founded in 1951 by \\ E. F. Beckenbach (1906-1982) and F. Wolf (1904-1989)
}

\section{EDITORS}

V. S. Varadarajan (Managing Editor)

Department of Mathematics

University of California

Los Angeles, CA 90095-1555

pacific@math.ucla.edu

Vyjayanthi Chari

Department of Mathematics

University of California

Riverside, CA 92521-0135

chari@math.ucr.edu

\section{Robert Finn}

Department of Mathematics Stanford University

Stanford, CA 94305-2125

finn@math.stanford.edu

Kefeng Liu

Department of Mathematics

University of California

Los Angeles, CA 90095-1555

liu@math.ucla.edu
Darren Long

Department of Mathematics

University of California

Santa Barbara, CA 93106-3080

long@math.ucsb.edu

Jiang-Hua Lu

Department of Mathematics

The University of Hong Kong

Pokfulam Rd., Hong Kong jhlu@maths.hku.hk

Alexander Merkurjev

Department of Mathematics

University of California

Los Angeles, CA 90095-1555

merkurev@math.ucla.edu
Sorin Popa

Department of Mathematics University of California

Los Angeles, CA 90095-1555 popa@math.ucla.edu

Jie Qing

Department of Mathematics

University of California

Santa Cruz, CA 95064

qing@cats.ucsc.edu

Jonathan Rogawski

Department of Mathematics

University of California

Los Angeles, CA 90095-1555

jonr@math.ucla.edu

\section{PRODUCTION}

pacific@math.berkeley.edu

\section{SUPPORTING INSTITUTIONS}

ACADEMIA SINICA, TAIPEI

CALIFORNIA INST. OF TECHNOLOGY INST. DE MATEMÁTICA PURA E APLICADA KEIO UNIVERSITY

MATH. SCIENCES RESEARCH INSTITUTE NEW MEXICO STATE UNIV.

OREGON STATE UNIV.

\author{
STANFORD UNIVERSITY \\ UNIV. OF BRITISH COLUMBIA \\ UNIV. OF CALIFORNIA, BERKELEY \\ UNIV. OF CALIFORNIA, DAVIS \\ UNIV. OF CALIFORNIA, LOS ANGELES \\ UNIV. OF CALIFORNIA, RIVERSIDE \\ UNIV. OF CALIFORNIA, SAN DIEGO \\ UNIV. OF CALIF., SANTA BARBARA
}

\author{
UNIV. OF CALIF., SANTA CRUZ \\ UNIV. OF MONTANA \\ UNIV. OF OREGON \\ UNIV. OF SOUTHERN CALIFORNIA \\ UNIV. OF UTAH \\ UNIV. OF WASHINGTON \\ WASHINGTON STATE UNIVERSITY
}

These supporting institutions contribute to the cost of publication of this Journal, but they are not owners or publishers and have no responsibility for its contents or policies.

See inside back cover or pacificmath.org for submission instructions.

The subscription price for 2012 is US \$420/year for the electronic version, and \$485/year for print and electronic.

Subscriptions, requests for back issues from the last three years and changes of subscribers address should be sent to Pacific Journal of Mathematics, P.O. Box 4163, Berkeley, CA 94704-0163, U.S.A. Prior back issues are obtainable from Periodicals Service Company, 11 Main Street, Germantown, NY 12526-5635. The Pacific Journal of Mathematics is indexed by Mathematical Reviews, Zentralblatt MATH, PASCAL CNRS Index, Referativnyi Zhurnal, Current Mathematical Publications and the Science Citation Index.

The Pacific Journal of Mathematics (ISSN 0030-8730) at the University of California, c/o Department of Mathematics, 969 Evans Hall, Berkeley, CA 94720-3840, is published monthly except July and August. Periodical rate postage paid at Berkeley, CA 94704, and additional mailing offices. POSTMASTER: send address changes to Pacific Journal of Mathematics, P.O. Box 4163, Berkeley, CA 94704-0163.

PJM peer review and production are managed by EditFLOW ${ }^{\mathrm{TM}}$ from Mathematical Sciences Publishers.

PUBLISHED BY PACIFIC JOURNAL OF MATHEMATICS

at the University of California, Berkeley 94720-3840

A NON-PROFIT CORPORATION

Typeset in LATEX

Copyright $(02012$ by Pacific Journal of Mathematics 


\section{PACIFIC JOURNAL OF MATHEMATICS}

Volume $258 \quad$ No. $2 \quad$ August 2012

Uniqueness theorems for $\mathrm{CR}$ and conformal mappings

YounG-JUn CHOI and JAE-CHEON JOO

Some finite properties for vertex operator superalgebras

269

CHONGYING DONG and JIANZHI HAN

On the geometric flows solving Kählerian inverse $\sigma_{k}$ equations

291

HAO FANG and MIJIA LAI

An optimal anisotropic Poincaré inequality for convex domains

GUOFANG WANG and CHAO XIA

Einstein metrics and exotic smooth structures

MASASHI ISHIDA

Noether's problem for $\hat{S}_{4}$ and $\hat{S}_{5}$

MING-CHANG KANG and JiAn ZHOU

Remarks on the behavior of nonparametric capillary surfaces at corners 369

KIRK E. LANCASTER

Generalized normal rulings and invariants of Legendrian solid torus links

MIKHAIL LAVROV and DAN RUTHERFORD

Classification of singular $\mathbb{Q}$-homology planes II: $\mathbb{C}^{1}$ - and $\mathbb{C}^{*}$-rulings.

KAROL PALKA

A dynamical interpretation of the profile curve of CMC twizzler surfaces

OSCAR M. PERDOMO

Classification of Ising vectors in the vertex operator algebra $V_{L}^{+}$

HIROKI SHIMAKURA

Highest-weight vectors for the adjoint action of $\mathrm{GL}_{n}$ on polynomials 